Pacific

Journal of

Mathematics

\title{
R-COVERED BRANCHED SURFACES
}

SANDRA SHIELDS 


\title{
R-COVERED BRANCHED SURFACES
}

\author{
SANDRA SHIELDS
}

\begin{abstract}
We give sufficient conditions for an R-covered codimension one foliation $\mathrm{F}$ of a closed 3-manifold to be carried by an $\mathrm{R}$ covered branched surface; that is, a branched surface carrying only foliations with the R-covered property. These conditions can be readily verified for many examples. In cases where the branched surface is generated by disks, the R-covered property is stable for $\mathrm{F}$ in the sense that all nearby foliations are also R-covered.
\end{abstract}

\section{Introduction}

In this paper, we study codimension one $C^{1}$ foliations of Riemannian 3manifolds. In particular, we examine foliations of a closed manifold $M$ that are covered by the canonical foliation of $\mathbf{R}^{3}$ by parallel hyperplanes, which we refer to as $\mathbf{R}$-covered. These foliations are particularly nice in the sense that they are completely determined by the induced action of $\pi_{1}(M)$ on the real line, which is the leaf space of the universal cover [So].

The R-covered property has been important in the study of foliations, especially those arising from Anosov flows. For example, if an Anosov foliation is $\mathbf{R}$-covered, then the other Anosov foliation associated with the flow also has this property and the Anosov flow can be shown to be transitive [So, Ba1, Ba2]. Certain restrictions on the manifold in combination with the $\mathbf{R}$-covered property have been used to show the Anosov flow is conjugate to a standard model, that is, a geodesic flow or a suspension of an Anosov diffeomorphism [P11, P12, Gh]. More recently, Fenley has used the $\mathbf{R}$-covered hypothesis to uncover the rich structure of metric and homotopy properties of flow lines in many Anosov flows [Fe1, Fe2]. He has also shown that each leaf in the lift of an incompressible $\mathbf{R}$-covered foliation (not necessarily Anosov) of a hyperbolic 3-manifold to the universal cover limits on all of $S_{\infty}^{2}[\mathrm{Fe} 3]$.

Foliations of a closed orientable manifold $M \neq S^{2} \times S^{1}$ that are $\mathbf{R}$-covered constitute a subset of the well-studied taut foliations. Tautness is a key to Roussarie's [Ro] and Thurston's [Th] results on isotoping incompressible tori and to Thurston's study of norm minimizing leaves. Gabai [Ga1, Ga2, Ga3] later used these results, by tautly foliating knot complements, to find the minimal genus spanning surface for a large class of knots and links. 
While tautness indicates the absence of Reeb components, it does not imply the $\mathbf{R}$-covered property. For example, there are many Anosov foliations that are not $\mathbf{R}$-covered. (See $[\mathbf{F r}-\mathbf{W} \mathbf{i}]$, [Bo-La].) Unlike the property of being taut, the $\mathbf{R}$-covered property is remarkably complex. In general, it is quite difficult to determine whether a foliation is $\mathbf{R}$-covered or not. It is known that foliations with a compact leaf $L$ are never $\mathbf{R}$-covered except when the ambient manifold fibers over $S^{1}$ with $L$ as fiber [Go-Sh]. However, when the foliation has no compact leaves, very little is known. One of the few things that is known is that foliations transverse to a non-Rcovered pseudo Anosov flow $\phi$ are $\mathbf{R}$-covered precisely when $\phi$ is regulating; there are, however, many examples of $\mathbf{R}$-covered Anosov flows transverse to $\mathbf{R}$-covered foliations that are not regulating [Fe5].

It is well-known that the property of being taut is stable in the sense that all foliations sufficiently close to a taut foliation (in the $C^{1}$ metric defined by Hirsch $[\mathbf{H i}]$ ) are also taut $[\mathbf{S u}]$. In contrast, the $\mathbf{R}$-covered property is much more delicate. There are simple examples demonstrating the $\mathbf{R}$-covered property is not stable in this sense. In fact, Calegari has described a way in which one could use shearing to perturb a foliation of a hyperbolic surface bundle over a circle to possibly obtain an arbitrarily close non-R-covered foliation $[\mathbf{C a}]$. While Calegari does not prove whether a non-R-covered foliation can result from such a shearing, his observations indicate that one might, and his example seems similar to one of Fenley's examples [Fe5] proving nonstability of the $\mathbf{R}$-covered property.

In this article, we find conditions that guarantee an $\mathbf{R}$-covered foliation $F$ of a closed orientable manifold $M \neq S^{2} \times S^{1}$ is carried by an $\mathbf{R}$-covered branched surface $W$ (i.e., a branched surface carrying only $\mathbf{R}$-covered foliations). If in addition to being $\mathbf{R}$-covered, $W$ is generated by disks, then we can apply a result in [Sh2] to obtain stability of the $\mathbf{R}$-covered property for all foliations carried by $W$. As a consequence, we say an $\mathbf{R}$-covered $W$ generated by disks is stably $\mathbf{R}$-covered.

We shall expand on results in [Go-Sh] in which a class of $\mathbf{R}$-covered branched surfaces was defined. (See Theorem 2.1.) Intuitively, these are branched surfaces that carry only taut foliations and do not contain the local behavior one would expect if there were a pair of leaves in the universal cover corresponding to a pair of nonseparable points in the leaf space. More specifically, a nonseparable pair of leaves in the universal cover gives rise to a smoothly immersed arc in the branched surface $W$ called a branching arc whose ends branch into smooth local subsets of $W$ from the same side. These arcs are discussed in Section 2 where we prove:

Theorem 2.2. Given a stably taut branched surface $W$ constructed from a foliation of a closed orientable 3-manifold $M \neq S^{2} \times S^{1}$, let $\widehat{W}$ be the lift of $W$ to the universal cover. Suppose there exists a finite set $\Gamma$ of branching 
arcs in $W$ such that the lift of any non-R-covered foliation carried by $W$ to a foliation carried by $\widehat{W}$ contains a pair of nonseparable leaves linked by a lift of some arc in $\Gamma$. For any $\mathbf{R}$-covered foliation $F$ carried by $W$, there exists a stably $\mathbf{R}$-covered branched surface $W^{\prime}$ also carrying $F$; in particular, the $\mathbf{R}$-covered property is stable for $F$.

The branched surface $W^{\prime}$ does not necessarily satisfy the hypothesis of Theorem 2.1. In particular, the class of stably $\mathbf{R}$-covered branched surfaces obtained here properly contains that which was described in [Go-Sh]. It is also worth noting that every $\mathbf{R}$-covered foliation is carried by a stably taut branched surface (defined in Section 2) [Go-Sh]. So the key hypothesis of Theorem 2.2 is the existence of the set $\Gamma$.

Corollary 2.3. Given a stably taut branched surface $W$ with disk sectors constructed from an $\mathbf{R}$-covered foliation $F$ of a closed orientable 3-manifold $M \neq S^{2} \times S^{1}$, let $\widehat{W}$ be the lift of $W$ to the universal cover $\widehat{M}$. If for every non-R-covered foliation carried by $W$ there is a branching arc contained in the boundary of some component of $\widehat{M}-\widehat{W}$ linking nonseparable leaves in the universal cover, then there exists a stably $\mathbf{R}$-covered branched surface $W^{\prime}$ carrying $F$ and the $\mathbf{R}$-covered property is stable for $F$.

In Section 3, we find verifiable conditions on the branch set $\mu$ of a branched surface $W$ constructed from an $\mathbf{R}$-covered foliation $F$ that guarantee the existence of an $\mathbf{R}$-covered branched surface carrying $F$. (See Theorem 3.2.) For many explicit examples, the $W$ we construct satisfies these conditions. In fact, it seems likely that all $\mathbf{R}$-covered foliations of orientable 3 -manifolds are carried by an $\mathbf{R}$-covered branched surface (not necessarily generated by disks). So while it is known that the $\mathbf{R}$-covered property is not always stable, there is often (perhaps always) a large class of $\mathbf{R}$-covered foliations near any $\mathbf{R}$-covered foliation $F$ of a closed manifold; specifically, there exists a transverse flow $\phi$ and a finite set $\Delta$ of compact integral surfaces of $F$ such that any foliation transverse to $\phi$ whose leaves contain the elements of $\Delta$ is R-covered.

The author would like to thank the referee for numerous suggestions that improved this paper.

\section{Branched surfaces constructed from foliations}

In this section we examine branched surfaces constructed from codimension one, transversely orientable foliations of an orientable 3-manifold $M$. These branched surfaces are in the class of regular branched surfaces introduced by R. Williams [Wi]. Since the construction, first suggested to S. Goodman by C. Danthony, is in an unpublished paper of Christy and Goodman [Ch-Go], we describe it here, including all details necessary for this article. 
Branched surface construction. We begin with a foliation $F$, a nonsingular flow $\phi$ transverse to $F$, and a generating set $\Delta=\left\{D_{i}\right\}$ of disjoint embedded compact surfaces with boundary (which is finite if the ambient manifold $M$ is closed), satisfying the following general position requirements:

(i) Each $D_{i}$ is embedded in a leaf of $F$ (hence is transverse to $\phi$ ).

(ii) Every orbit of $\phi$ meets the interior of some element of $\Delta$ in forward and backward time.

(iii) For every $i_{0}$, the set of points in $\operatorname{Bdy}\left(D_{i_{0}}\right)$ whose orbit under $\phi$ (or $\left.\phi^{-1}\right)$ meets $\cup \operatorname{Bdy}\left(D_{i}\right)$ before meeting $\cup \operatorname{Int}\left(D_{i}\right)$ is finite.

(iv) Any orbit of $\phi$ meets $\cup \operatorname{Bdy}\left(D_{i}\right)$ at most twice.

It is worth noting that we can always choose a generating set $\Delta$ consisting of embedded disks. For example, cover $M$ with foliation boxes for $F$ and let $\Delta$ contain a slice from each box. Then modify each slice slightly so that $\Delta$ satisfies the general position requirements above.

We cut $M$ open along the interior of each element of $\Delta$ to obtain a submanifold $M^{*}$ which can be embedded in $M$ so that its boundary contains $\cup \operatorname{Bdy}\left(D_{i}\right)$. This can be thought of as blowing air into the leaves of $F$ to create an air pocket at each element of the generating set. By requirement (ii) above, the restriction of $\phi$ to $M^{*}$ is a flow $\phi^{*}$ with the property that each orbit is homeomorphic to the unit interval $[0,1]$. We next form a quotient space by identifying points that lie on the same orbit of $\phi^{*}$. That is, we take the quotient $M^{*} / \sim$, where $x \sim y$ if $x$ and $y$ lie on the same interval orbit of $\phi^{*}$. We may think of this as enlarging the components of $M-M^{*}$ until each interval orbit of $\phi^{*}$ is contracted to a point in $M$. The embedded copy of the resulting quotient space is the branched surface $W$; it is called the branched surface corresponding to $(F, \phi, \Delta)$. By its construction, $W$ can be made transverse to $\phi$. The general position requirements for $\Delta$ imply that $W$ is a connected 2-dimensional complex with a set of charts defining local orientation preserving diffeomorphisms onto one of the models in Figure 1.1, such that the transition maps are smooth and preserve transverse orientation indicated by the arrows. Each local model projects horizontally onto a vertical model of $\mathbf{R}^{2}$. So $W$ has a smooth structure induced by $T \mathbf{R}^{2}$ when we pull back each local projection.

A branched surface constructed from a foliation shall be a branched surface embedded in the ambient manifold and obtained in the above fashion using some transverse flow $\phi$. Each is a connected 2-manifold except on a dimension one subset $\mu$ called the branch set. The elements of $\Delta$ can be assumed to be chosen large enough to ensure that $\mu$ is connected. The set $\mu$ is a 1-manifold except at isolated points called crossings where it intersects itself transversely. (There are only finitely many of these points when $M$ is closed.) Each component of $W-\mu$ is called a sector of $W$. 

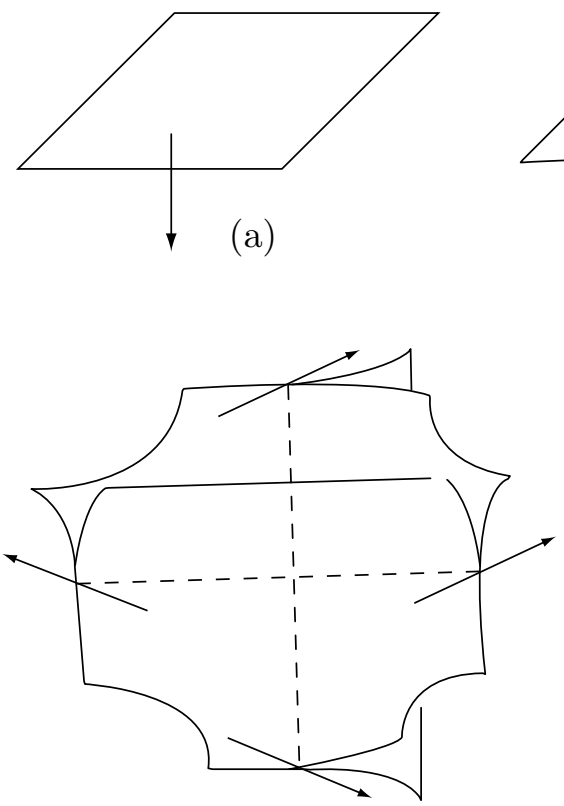

(c)
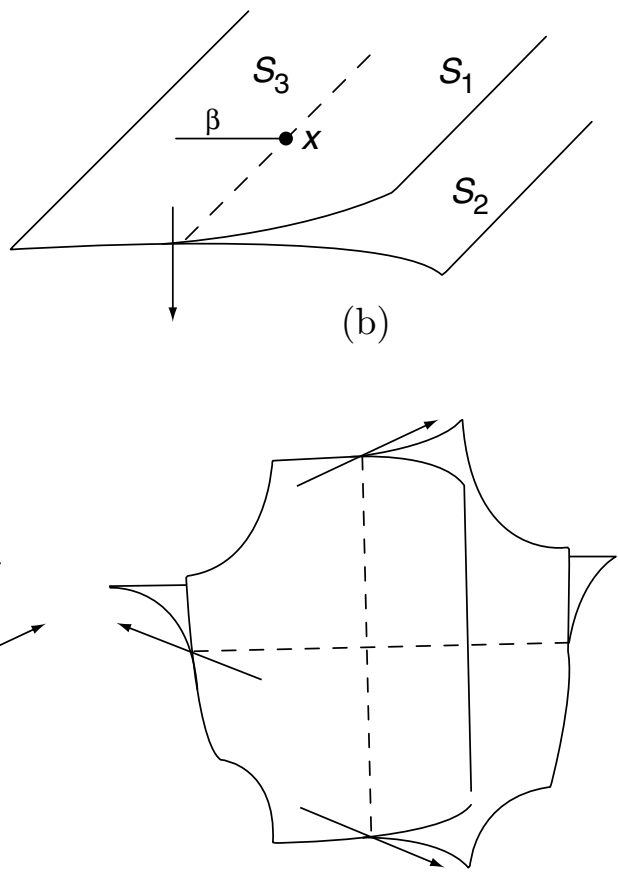

(d)

Figure 1.1.

Curves in the branched surface. Formally, a curve in $M$ is a continuous map from a connected subset of $\mathbf{R}$ into $M$. However, we consider a curve to be the image of such a map, where the map parameterizes the curve. We say the curve is immersed (embedded) if this map is an immersion (embedding respectively.) For example, a curve immersed in a branched surface $W \subseteq M$ is the image of an immersion from a connected subset of $\mathbf{R}$ into $W$. The beginning and end of a curve refer to the negative and positive boundary, respectively, induced by the parameterization. For the sake of simplicity, we consider two curves $\gamma$ and $\gamma^{\prime}$ to be the same if $\gamma(t)=\gamma^{\prime}(t)$ after some orientation preserving reparameterization of each. We say a curve $\gamma^{\prime}$ is contained in a second curve $\gamma$ (or more simply $\gamma^{\prime}$ is in $\gamma$ ) only if $\gamma^{\prime}$ is a subarc of $\gamma$. That is, there exist curves $\alpha_{1}$ and $\alpha_{2}$ such that $\gamma$ is equal to the composition $\alpha_{2} * \gamma^{\prime} * \alpha_{1}$. A loop in $\gamma$ will be a subarc of $\gamma$ with nonempty interior that begins and ends at the same point. A smooth arc in $W$ is an arc contained in $W$ that is smooth under the structure inherited from $W$. Throughout, we shall not consider curves in $W$ for which some subarc is homotopic, through a sector of $W$, to an arc in the branch set containing no crossings. 
Given a point $x$ contained in the branch set $\mu$ of $W$ that is not a crossing, there are locally 3 sectors, $S_{1}, S_{2}$, and $S_{3}$ that are adjacent at $x$ such that $\operatorname{cl}\left(S_{1}\right) \cup S_{3}$ and $\operatorname{cl}\left(S_{2}\right) \cup S_{3}$ are smooth submanifolds of $W$ (i.e., the set of charts locally defines a smooth immersion into a planar subset of $\mathbf{R}^{3}$ on $\operatorname{cl}\left(S_{1}\right) \cup S_{3}$ and $\left.\operatorname{cl}\left(S_{2}\right) \cup S_{3}\right)$. Assume that in a local neighborhood of $x$, forward orbits under $\phi$ of points in $S_{1}$ flow into $S_{2}$. For any arc $\alpha$ immersed in $W$ that is transverse to $\mu$ at $x$, we can choose a subarc $\beta$ of $\alpha$ that has nonempty interior and meets $\mu$ only at $x$. We say $S_{2}$ is an upper branch along $\alpha$ if $\beta \cap S_{2}=\varnothing$ for any choice of $\beta$. See Figure 1.1(b). Similarly, $S_{1}$ is a lower branch along $\alpha$ if $\beta \cap S_{1}=\varnothing$ for any choice of $\beta$. A branch along $\alpha$ is an incoming sector branching into $\alpha$ at $x$ if there is a subarc $\beta$ of $\alpha$ (chosen as above) that either ends at $x$ and is contained in $S_{1} \cup S_{2} \cup\{x\}$ or begins at $x$ and is contained in $S_{3} \cup\{x\}$. Likewise, a branch along $\alpha$ is an outgoing sector branching from $\alpha$ at $x$ if there is a subarc $\beta$ as above that either ends at $x$ and is contained in $S_{3} \cup\{x\}$ or begins at $x$ and is contained in $S_{1} \cup S_{2} \cup\{x\}$.

We can extend the notion of incoming and outgoing sectors to a curve $\alpha$ immersed in the branch set $\mu$ of $W$. For each crossing $x$ of $\mu$ contained in $\alpha$, we can choose a subarc $\beta$ of $\alpha$ that is bounded by $x$ and contains no other crossings of $\mu$. (Each time int $(\alpha)$ meets $x, \beta$ can be chosen to either begin or end at $x$.) There is precisely one sector $S$ whose boundary is transverse to $\beta$ at $x$. If the subarc $\beta$ can be chosen to begin (end) at $x$ and the union of $\operatorname{cl}(S)$ with those sectors whose boundaries both contain $x$ and do not intersect int $(\beta)$ is smooth, then $S$ is an outgoing (incoming, respectively) sector branching from $\alpha$. For example, in both Figures 1.2(a) and 1.2(b), a curve $\beta$ is indicated by the directed arcs. The corresponding sector $S$ for each is shaded. In Figure 1.2(a), $S$ is a lower outgoing sector branching from any $\alpha$ containing $\beta$, whereas in Figure 1.2(b) the sector $S$ is not a lower outgoing sector along any such $\alpha$. In the case where $\alpha$ ends (begins) at $x$, the sector $S$ is an outgoing (incoming, respectively) sector branching from $\alpha$ if for some subarc $\beta$ chosen as above, the sector $S$ is an outgoing (incoming) sector branching from a smooth curve in $\mu$ that contains $\beta$ and meets no crossings other than $x$. For example, the sector $S$ in Figure 1.2(b) is a lower incoming sector along any $\alpha$ beginning with $\beta$.

Foliations carried by a branched surface. Note that if we thicken the branched surface $W$ in the transverse direction to recover the interval orbits of $\phi^{*}$, we retrieve $M^{*}$ which, for that reason, we shall henceforth call $N(W)$, the neighborhood of $W$. Also note that the boundary of $N(W)$ is an embedded closed surface which has a smooth structure except along curves corresponding to the boundaries of the generating surfaces.

Throughout, $\pi: N(W) \rightarrow W$ will denote the quotient map which identifies points in the same orbit of $\phi^{*}$. We say the image $x$ of a point under 


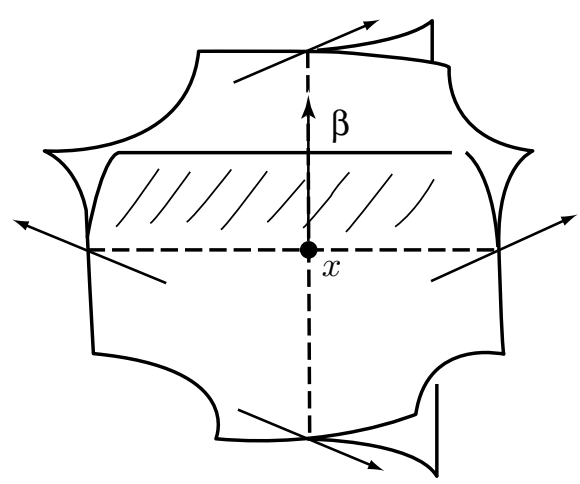

(a)

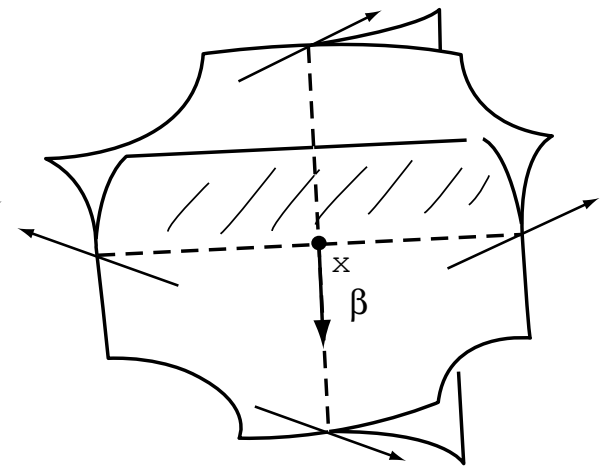

(b)

Figure 1.2.

Local $W$

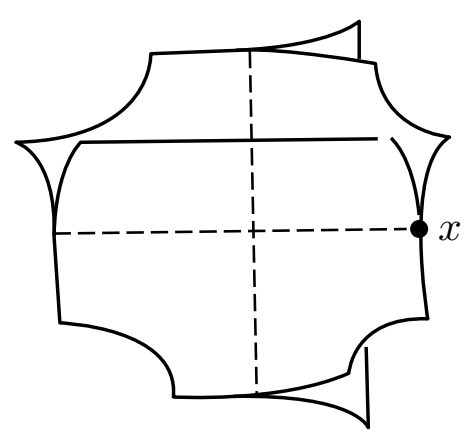

Thicken to local $N(W)$ :

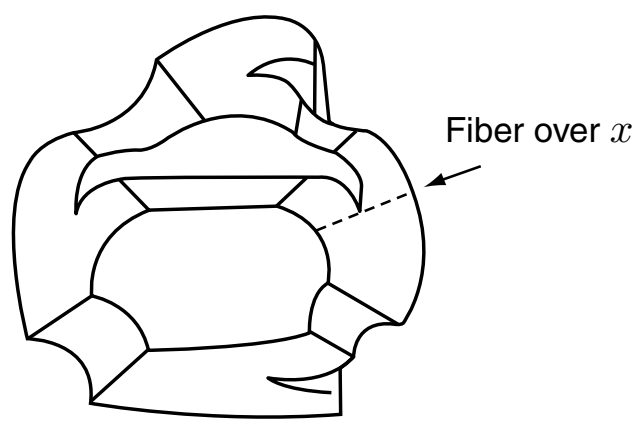

Figure 1.3.

this map is the projection of that point. Accordingly, we say points in the preimage of $x$ lie over $x$. In particular, the interval orbit of $\phi^{*}$ that projects onto $x$ will be referred to as the fiber of $N(W)$ over $x$. (See Figure 1.3.)

A foliation $F$ transverse to $\phi$ clearly gives rise to a foliation of $N(W)$, which we shall also denote by $F$, with leaves transverse to the fibers of $N(W)$. Each boundary component of $\mathrm{N}(\mathrm{W})$ is contained in a leaf of this foliation. These leaves containing the boundary components of $N(W)$ are precisely the (cut-open) leaves of the original foliation containing the elements of $\Delta$. (They can be thought of as leaves of the original foliation with air blown into them.) Figure 1.4 shows a local picture of the foliation of $N(W)$.

There are, of course, many foliations like $F$ that are transverse to the fibers of $N(W)$ with the property that each boundary component of $N(W)$ is contained in a leaf. When we collapse the components of $M-N(W)$ (i.e., the air pockets), each of these foliations of $N(W)$ yields a foliation of $M$, 


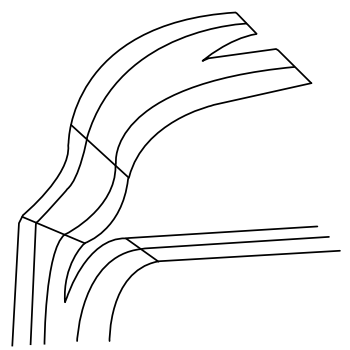

Figure 1.4.

also transverse to $\phi$, which we say is carried by $W$. Indeed, $W$ carries a foliation $G$ if and only if $W$ corresponds to $(G, \phi, \Delta)$.

In what follows, $W$ will be a branched surface constructed from a foliation $F$ of a closed manifold $M$ as described above, and $\widehat{W}$ will be the lift of $W$ to the universal cover $\widehat{M}$. In other words, if $W$ corresponds to $(F, \Delta, \phi)$, then $\widehat{W}$ is the branched surface corresponding to $(\widehat{F}, \widehat{\Delta}, \widehat{\phi})$, where $\widehat{F}, \widehat{\Delta}$ and $\widehat{\phi}$ represent the lifts of $F, \Delta$, and $\phi$, respectively, to the universal cover. The covering map $\rho_{M}$ of $M$ by $\widehat{M}$ acts on $N(\widehat{W})$ and induces a covering map $p: \widehat{W} \rightarrow W$ such that $\pi \circ \rho_{M}=\rho \circ \widehat{\pi}$, where $\widehat{\pi}$ is the quotient map from $N(\widehat{W})$ onto $\widehat{W}$. (Details are given in $[\mathbf{S h} \mathbf{1}]$.)

\section{Stability of the R-covered property}

Throughout, $F$ will be a codimension one $C^{1}$ foliation of a closed orientable 3-manifold $M \neq S^{2} \times S^{1}$. Passing to a double cover of $M$ if necessary, we may assume that $F$ is transversely orientable.

If $W$ is a branched surface constructed from a foliation $F$ whose generating set $\Delta$ consists of embedded disks, then all foliations sufficiently close to $F$ are also carried by $W$ [Sh2]. (Here we are using the $C^{1}$ metric defined in $[\mathbf{H i}]$, where a nearby foliation is obtained by perturbing the tangent bundle to the leaves to another integrable plane field.) In particular, if a branched surface $W$ is generated by disks and every foliation carried by $W$ has a certain topological property, then we know that property is stable for all foliations carried by $W$.

Here we give conditions that guarantee a branched surface $W$ constructed from a foliation is $\mathbf{R}$-covered; that is, $W$ has the property that every foliation carried by it is $\mathbf{R}$-covered. If a branched surface $W$ is $\mathbf{R}$-covered and generated by disks, then we say it is stably $\mathbf{R}$-covered since any foliation $F$ carried by that $W$ is $\mathbf{R}$-covered and this property is stable for each such $F$ (in the sense that all foliations sufficiently close to $F$ are also $\mathbf{R}$-covered). We realize that this latter terminology is slightly misleading. That is, a branched 
surface could carry only foliations for which the $\mathbf{R}$-covered property is stable without being generated by disks. However, our stability results rely on the branched surface being generated by disks. So for simplicity in our discussion, we define stably $\mathbf{R}$-covered as above.

Every $\mathbf{R}$-covered foliation of a closed orientable manifold $M \neq S^{2} \times S^{1}$ is taut; i.e., there exists a positive transverse arc from any leaf to any other leaf. (For details, see Lemma B [Go-Sh].) So we first review the characterization of branched surfaces carrying only taut foliations.

Clearly any leaf in a foliation carried by a branched surface $W$ projects onto several sectors of $W$ and each sector has a transverse orientation, which it inherits from the leaves. So to ensure tautness, it suffices that there exists a positive transversal to $W$ between any two sectors. When we regard the branched surface as a simplicial complex, this translates to a condition on the dual graph. More precisely, we define the dual graph to the branched surface $W$ as follows: each component of $M-W$ will contain one vertex. There will be an edge through each sector of $W$, oriented according to the transverse orientation of $W$, joining two vertices. In particular, the dual graph is a directed graph in $M$ that is transverse to $W$. If for any ordered pair of vertices $(v, w)$ of the dual graph there is a positively oriented path from $v$ to $w$, then the dual graph is said to be transitive. When a foliation is carried by a branched surface with a transitive dual, then there is a positively oriented transverse arc from any leaf to any other leaf; specifically, there exists a positively oriented transverse loop through each leaf, so the foliation is taut. The converse is also true. Indeed, any branched surface constructed from a taut foliation using a volume-preserving transverse flow has a transitive dual. So the transitive dual property characterizes branched surfaces carrying only taut foliations [Go-Sh]. Consequently, if $W$ has a transitive dual we say it is a taut branched surface. If, in addition, $W$ is generated by disks, we say $W$ is stably taut.

Now suppose $W$ is a taut branched surface corresponding to $(F, \Delta, \phi)$, where the surfaces in the generating set $\Delta$ are not necessarily embedded disks. In this case, there is a transverse loop through every leaf of $F$, which ensures the absence of Reeb components. This, in turn, precludes vanishing cycles. So when we lift $F$ to the universal cover, we obtain a foliation $\widehat{F}$ of $\mathbf{R}^{3}$ where all the leaves are topologically closed planes (since $M$ is assumed to be orientable and not homeomorphic to $S^{2} \times S^{1}$ ) and the leaf space is a 1-manifold, possibly non-Hausdorff [Ha]. At this stage, the only obstruction to $F$ being $\mathbf{R}$-covered is the existence of two leaves in the universal cover which are nonseparable in the leaf space; in other words, a pair of leaves $\widehat{A}$ and $\widehat{B}$ in $\widehat{F}$ which do not have disjoint saturated neighborhoods. In particular, there is a 1-parameter family $\left\{\widehat{K}_{n}\right\}$ of leaves, parameterized by $n \in I$ (where $I$ is the real interval $(0,1)$ ), which lie on the same side of 


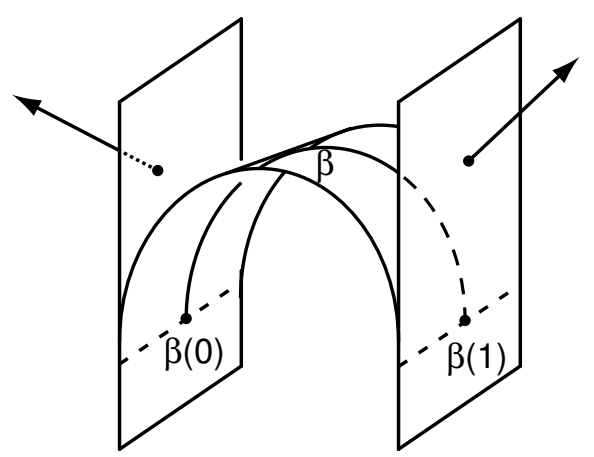

Figure 2.1.

$\widehat{A}$ and $\widehat{B}$ and monotonically approach both $\widehat{A}$ and $\widehat{B}$ in the leaf space as $n \rightarrow 1$. (We assume points in the leaf space are ordered according to the transverse orientation of $\widehat{F}$ induced by the transverse flow $\widehat{\phi}$ ). This sort of local behavior occurs, for example, in the lifts of certain Anosov foliations, like the one constructed in $[\mathbf{F r}-\mathbf{W} \mathbf{i}]$. We can choose points $\widehat{x}_{n}, \widehat{y}_{n}$ in $\widehat{K}_{n}$ for each $n$, such that $\left\{\widehat{x}_{n}\right\}$ converges to a point $\widehat{x}$ in $\widehat{A}$ along an orbit of $\widehat{\phi}$ (or $\widehat{\phi}^{-1}$ ) and $\left\{\widehat{y}_{n}\right\}$ converges to a point $\widehat{y}$ in $\widehat{B}$ along an orbit of $\widehat{\phi}\left(\widehat{\phi}^{-1}\right.$, respectively). Now for every $N \in(0,1)$ the orbit from $\widehat{x}_{N}$ to $\widehat{x}$ is finite, so there are at most finitely many points along this orbit that are contained in a generating surface for $\widehat{W}$. (If not, such points would accumulate along this orbit on some point $\widehat{z}$. However the generating set for $W$ is finite and each element is compact. So if we consider the point $z$ covered by $\widehat{z}$, the intersection of all generating surfaces for $W$ with any evenly covered neighborhood $U$ of $z$ has finitely many components. Furthermore, we can choose $U$ small enough so that any orbit of $\left.\phi\right|_{U}$ intersects each of these components in at most one point, a contradiction.) So if each $\widehat{K}_{n}$ lies on the negative (positive) side of $\widehat{A}$ and $\widehat{B}$, then we can choose $N$ large enough so that orbits of $\widehat{\phi}\left(\widehat{\phi}^{-1}\right.$ respectively) from $\widehat{x}_{N}$ to $\widehat{A}$ and from $\widehat{x}_{N}$ to $\widehat{B}$ do not meet any generating surface for $\widehat{W}$. These orbits are, up to orientation, contained in fibers of $N(\widehat{W})$, so the local picture in $\widehat{W}$ is as shown in Figure 2.1.

More precisely, if $\widehat{F}$ contains a pair of leaves $\widehat{A}$ and $\widehat{B}$ that are nonseparable on their negative (positive) sides, then there is a smoothly embedded arc $\widehat{\beta}$ in $\widehat{W}$ transverse to $\widehat{\mu}$ and containing no crossings of $\widehat{\mu}$ with the property that the ends of $\widehat{\beta}$ branch into the negative (positive) side of two smooth local subsets of $\widehat{W}$. In particular, the arc $\widehat{\beta}$ has an upper (lower) outgoing sector branching from its initial point and an upper (lower) incoming sector branching into its terminal point. Any arc embedded in $\widehat{W}$ with these properties is called a negatively (positively) branching arc in $\widehat{W}$. Each branching 
$\operatorname{arc} \widehat{\beta}$ in $\widehat{W}$ covers a branching arc $\beta$ in $W$. A branching arc corresponding to a nonseparable pair of leaves $\widehat{A}$ and $\widehat{B}$ as above can be chosen so that its ends lie in $\widehat{\pi}(\widehat{A})$ and $\widehat{\pi}(\widehat{B})$, respectively, and some integral curve $\widehat{\kappa}$ of $\widehat{F}$ lies over it in $N(\widehat{W})$. In this case, we say $\widehat{\beta}$ is a branching arc linking $\widehat{A}$ and $\widehat{B}$.

If the branched surface $\widehat{W}(W)$ has only disk sectors, then certain branching arcs in $\widehat{W}(W$, respectively) can be regarded as essentially the same. In particular, the boundary of each disk sector $S$ is contained in the branch set and can be partitioned by crossings into disjoint open arcs. Any two curves whose interiors lie in $S$ are equivalent if their initial points both lie in the interior of $S$ or in the same interval of the partition, and if the same condition holds for their terminal points. Now, any finite topologically closed curve immersed in $\widehat{W}(W)$ that is transverse to the branch set is a composition of finitely many curves whose interiors are contained in the disk sectors of $\widehat{W}(W$, respectively). Two such curves are equivalent if they contain no crossings of the branch set and are piecewise equivalent. It is worth emphasizing that this definition applies only to curves contained in branched surfaces with disk sectors. Also, note that if $\widehat{\beta}$ is a branching arc in $\widehat{W}$ linking nonseparable leaves $\widehat{A}$ and $\widehat{B}$, and $\widehat{\beta}^{\prime}$ is equivalent to $\widehat{\beta}$, then $\widehat{\beta}^{\prime}$ is also a branching arc linking $\widehat{A}$ and $\widehat{B}$.

Now let $W$ be a taut branched surface carrying a foliation $F$ that has sectors of any type and is not necessarily generated by disks. Although nonseparable leaves in the lift $\widehat{F}$ of $F$ to the universal cover give rise to branching arcs in a branched surface $\widehat{W}$ carrying $\widehat{F}$, not every branching arc in $\widehat{W}$ indicates a pair of nonseparable leaves. In fact, any smoothly embedded integral curve of $\widehat{F}$ whose interior is contained in an element of $\widehat{\Delta}$ (generating $\widehat{W}$ ) and whose endpoints are in the boundary of that generating surface has two images in $\widehat{W}$, both of which are branching arcs. So branching arcs also occur in branched surfaces carrying only $\mathbf{R}$-covered foliations. Certain curves embedded in $\widehat{W}$, called bypasses, offer a topological means for identifying those branching arcs that cannot link nonseparable leaves. Specifically, a smoothly embedded arc $\widehat{\delta}$ in $\widehat{W}$ transverse to $\widehat{\mu}$ and fixed point homotopic to a negatively branching arc $\widehat{\beta}$ is a bypass for $\widehat{\beta}$ if it contains no crossings of $\widehat{\mu}$ or negatively branching arcs. (Figure 2.2 shows a bypass for a branching arc $\widehat{\beta}$.) A bypass for a positively branching arc is defined in an analogous manner. Clearly a bypass $\widehat{\delta}$ for a branching arc $\widehat{\beta}$ in $\widehat{W}$ covers an immersed arc $\delta$ homotopic to $\beta$ (the branching arc covered by $\widehat{\beta})$ with the same properties. Accordingly, such an $\operatorname{arc} \delta$ is a bypass for $\beta$. We say that a branching arc $\beta$ in a taut branched surface $W$ (as above) is critical if it lifts to an arc linking nonseparable leaves in some foliation carried by $\widehat{W}$. In particular, critical branching arcs have no bypasses. For 


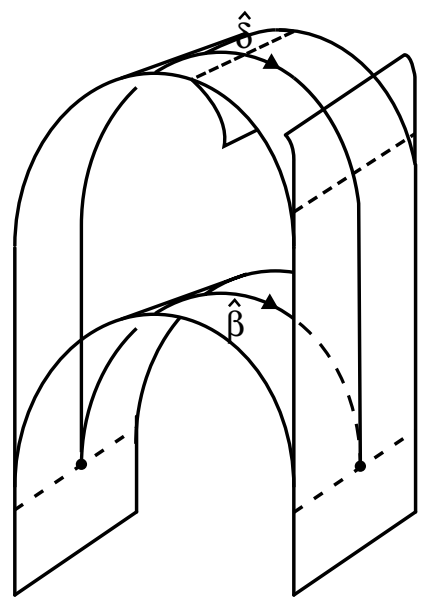

Figure 2.2.

suppose, to the contrary, that a branching arc $\widehat{\beta}$ has a bypass $\widehat{\delta}$ yet links leaves $\widehat{A}$ and $\widehat{B}$ which are nonseparable on, say, their negative sides. If the projection of $\widehat{A}$ branches away from $\widehat{\delta}$ along an upper outgoing sector at some point $\widehat{x}$ in $\widehat{\delta}$, then there are no upper incoming sectors branching into $\widehat{\delta}$ after $\widehat{x}$. In this case, there exists a positive transversal from $\widehat{B}$ to $\widehat{A}$, a contradiction. So $\widehat{\pi}(\widehat{A})$ branches away from $\widehat{\delta}$ along a lower outgoing sector at some point $\widehat{x}$ in $\widehat{\delta}$. Similarly, we find $\widehat{\pi}(\widehat{B})$ branches into $\widehat{\delta}$ along a lower incoming sector at some point after $\widehat{x}$ in $\widehat{\delta}$; more precisely, $\widehat{\delta}$ contains a positively branching arc with $\widehat{\pi}(\widehat{A})$ and $\widehat{\pi}(\widehat{B})$ branching from its respective ends. However, the uppermost leaf whose projection meets this arc is cut by positive transversals (not necessarily fibers) from $\widehat{A}$ and $\widehat{B}$, contradicting our assumption that $\widehat{A}$ and $\widehat{B}$ are nonseparable on their negative sides. So if a foliation $F$ is carried by a taut branched surface $W$ and every branching arc has a bypass, then $F$ is $\mathbf{R}$-covered. If, in addition, $W$ is stably taut (hence, is generated by disks), then the $\mathbf{R}$-covered property is stable for $F$.

These observations lead to the following:

Theorem 2.1 ([Go-Sh]). Given a stably taut branched surface $W$ constructed from a foliation of a closed orientable 3 -manifold $M \neq S^{2} \times S^{1}$, if every branching arc has a bypass, then $W$ is $\mathbf{R}$-covered and the $\mathbf{R}$-covered property is stable for each foliation $F$ carried by $W$; in particular, $F$ and all foliations sufficiently close to $F$ are $\mathbf{R}$-covered.

If a foliation $F$ is $\mathbf{R}$-covered, then we can construct a branched surface $W$ from it using a generating set consisting of embedded disks and a transverse flow that is volume-preserving. As noted earlier, $W$ will be stably taut. However, such a $W$ will often contain branching arcs without bypasses. 
In [Go-Sh], a technique was given for modifying $W$ to produce bypasses for certain branching arcs. Unfortunately, this can create annuli in the generating set. So while $W$ will still be taut after these modifications, it might not be stably taut. Moreover, application of this technique frequently does not produce a branched surface where every branching arc has a bypass. One obstruction is that it can only be applied to branching arcs that are contained in the projection of an integral curve of $F$. Another is that most branched surfaces contain infinitely many branching arcs. For example, an infinite set of branching arcs may share a nontrivial loop and differ only in the number of times this loop is traversed. (Here a loop in a branching arc is a subarc with nonempty interior that begins and ends at the same point.)

Consequently, we shall weaken the hypotheses of Theorem 2.1 significantly. To prove stability of the $\mathbf{R}$-covered property under these new conditions, we modify $W$ using a different technique that will not create annuli in the generating set.

The main result is as follows:

Theorem 2.2. Given a stably taut branched surface $W$ constructed from a foliation of a closed orientable 3-manifold $M \neq S^{2} \times S^{1}$, let $\widehat{W}$ be the lift of $W$ to the universal cover. Suppose there exists a finite set $\Gamma$ of branching arcs in $W$ such that the lift of any non-R-covered foliation carried by $W$ to a foliation carried by $\widehat{W}$ contains a pair of nonseparable leaves linked by a lift of some arc in $\Gamma$. For any $\mathbf{R}$-covered foliation $F$ carried by $W$, there exists a stably $\mathbf{R}$-covered branched surface $W^{\prime}$ also carrying $F$; in particular, the $\mathbf{R}$-covered property is stable for $F$.

Proof. Suppose $W$ and $\Gamma$ are as in the hypotheses. In particular, $W$ is stably taut, so it is generated by disks and all foliations carried by $W$ are taut. Let $F$ be an $\mathbf{R}$-covered foliation carried by $W$ and let $\beta$ be any critical branching arc in $\Gamma$. Without loss of generality, we may assume $\beta$ is negatively branching. Substituting a homotopic branching arc if necessary, we may also assume that $\beta$ does not begin and end at the same point; that is, $\beta(0) \neq \beta(1)$. We first consider the case where $\beta$ and its lift $\widehat{\beta}$ to $\widehat{W}$ are projections of integral curves of $F$ and $\widehat{F}$ respectively. In particular, $\widehat{\beta}$ is contained in $\widehat{\pi}(\widehat{L})$ for some leaf $\widehat{L}$ of $\widehat{F}$.

Since $\widehat{\beta}$ has no bypass, the positive ends of the fibers over $\widehat{\beta}(0)$ and $\widehat{\beta}(1)$ lie in distinct boundary components of $N(\widehat{W})$ which correspond respectively to elements $\widehat{D}_{0}$ and $\widehat{D}_{1}$ of $\widehat{\Delta}$. Let $\widehat{L}_{0}$ and $\widehat{L}_{1}$ be the leaves of $\widehat{F}$ containing $\widehat{D}_{0}$ and $\widehat{D}_{1}$ respectively. In the natural ordering of the leaves of $\widehat{F}$ (induced by a copy of the leaf space $\mathbf{R}$ which, in turn, is oriented according to the direction of the transverse flow $\widehat{\phi}$ ), we have either:
(i) $\widehat{L}_{0}<\widehat{L}_{1}$,
(ii) $\widehat{L}_{0}>\widehat{L}_{1}$, or
(iii) $\widehat{L}_{0}=\widehat{L}_{1}$. 

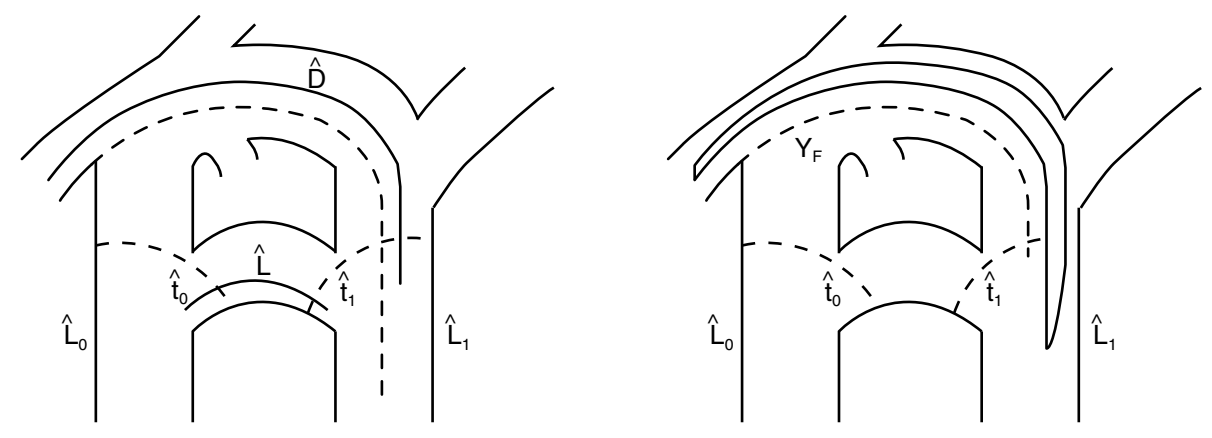

Figure 2.3.

In case (i), $\widehat{L}<\widehat{L}_{0}<\widehat{L}_{1}$, and since the fiber $\widehat{t}_{1}$ over $\widehat{\beta}(1)$ meets $\widehat{L}$ and $\widehat{L}_{1}$, it meets all leaves between $\widehat{L}$ and $\widehat{L}_{1}$. It follows that $\widehat{t}_{1}$ also meets $\widehat{L}_{0}$, so we can find a curve $\widehat{\gamma}_{F}$ smoothly embedded in $\widehat{L}_{0}$ that begins in $\partial \widehat{D}_{0}$ and cuts the fiber $\widehat{t}_{1}$. When choosing $\widehat{\gamma}_{F}$, we shall want to ensure that its quotient $\gamma_{F}$ does not cut $t_{0}$ or $t_{1}$ more than once: i.e., we choose $\widehat{\gamma}_{F}$ so it descends to a smoothly immersed integral curve $\gamma_{F}$ of $F$ whose interior does not meet $t_{0}$ or $t_{1}$. We can then find a disk $\widehat{D}$ embedded in a leaf of $\widehat{F}$ that does not intersect any generating disks for $\widehat{W}$ and lies sufficiently close to $\widehat{D}_{0}$ on the positive side so that all orbits from $\widehat{\gamma}_{F}$ meet $\widehat{D}$ before meeting a generating disk. Adding $\widehat{D}$ to the generating set $\widehat{\Delta}$ (and its quotient $D$ to $\Delta$ ) changes the branched surface by splitting it along an embedded disk containing $\widehat{\pi}\left(\widehat{\gamma}_{F}\right)\left(\pi\left(\gamma_{F}\right)\right.$ respectively). By the way we chose $\widehat{\gamma}_{F}$, we can ensure no covering translate of $\widehat{D}$ cuts $\widehat{t}_{0}$ or the new fiber $\widehat{t}_{1}^{*}$ over $\widehat{\beta}(1)$. So after this modification, there is a smoothly embedded arc $\widehat{\delta}$ in $\widehat{W}$ containing $\widehat{\pi}\left(\widehat{\gamma}_{F}\right)$ which is fixed point homotopic to $\widehat{\beta}$ and has only one upper branch along its interior at the initial point of $\widehat{\pi}\left(\widehat{\gamma}_{F}\right)$. (See Figure 2.3.) In this way, we create bypasses $\widehat{\delta}$ and $\delta$ for $\widehat{\beta}$ and $\beta$ respectively.

Cases (ii) and (iii) are analogous. (In case (iii), the curve $\widehat{\delta}$ that we create has an upper incoming sector branching into it at $\widehat{\pi}\left(\widehat{\gamma}_{F}(0)\right)$ and an upper outgoing sector branching from it at $\widehat{\pi}\left(\widehat{\gamma}_{F}(1)\right)$, but contains no negatively branching arcs.)

So whenever a critical branching arc $\beta$ is the projection of an integral curve of $F$, we can modify $W$ to create a bypass $\delta$ for $\beta$. If, on the contrary, $\beta$ is not the projection of an integral curve of $F$, then it can be destroyed. Reversing the orientation of $\beta$ if necessary, there exists an integral curve $\gamma_{F}(t)_{0 \leq t \leq 1}$ of $F$ beginning in the intersection of $\partial N(W)$ with the interior of a fiber over int $(\beta)$ such that for some $\varepsilon>0, \pi\left(\gamma_{F}(t)\right)_{0 \leq t \leq 1-\varepsilon}$ is contained in $\beta, \beta \cap \pi\left(\gamma_{F}(t)\right)_{1-\varepsilon<t \leq 1}=\varnothing$ and $\gamma_{F}(1-\varepsilon) \notin \partial N(W)$. See Figure 2.4. So there exist incoming and outgoing branches along $\beta$ at $\pi\left(\gamma_{F}(0)\right)$ and $\pi\left(\gamma_{F}(1-\varepsilon)\right)$ 


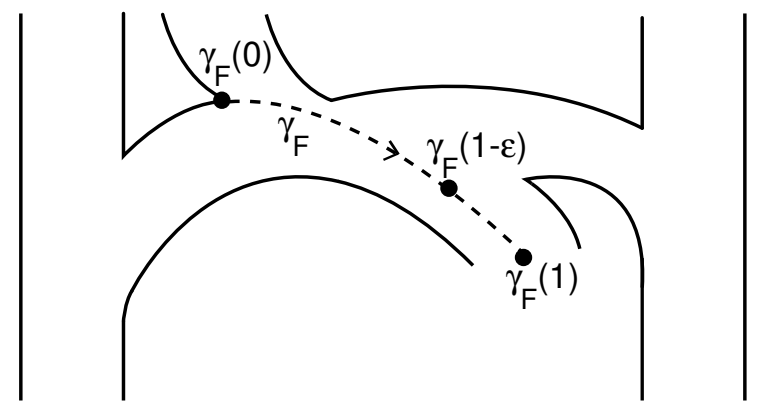

Figure 2.4.

respectively. Moreover, we can choose $\gamma_{F}$ so that if the sector branching into $\beta$ at $\pi\left(\gamma_{F}(0)\right)$ is an upper (lower) incoming sector, then the sector branching from $\beta$ at $\pi\left(\gamma_{F}(1-\varepsilon)\right)$ is a lower (upper, respectively) outgoing sector containing $\pi\left(\gamma_{F}(t)\right)_{1-\varepsilon<t \leq 1}$. (For details, see [Sh1].) Clearly, the initial point of $\gamma_{F}$ lies in the boundary of some generating disk $D_{0}$ as before.

If there is an upper (lower) sector branching into $\beta$ at $\pi\left(\gamma_{F}(0)\right)$, we may add an embedded disk $D$ to the generating set which lies sufficiently close to $D_{0}$ on the positive (negative) side so that all forward (backward, respectively) orbits from $\gamma_{F}$ meet $D$ before meeting any other generating disk. This change in $\Delta$ corresponds to splitting $W$ along a disk containing $\pi\left(\gamma_{F}\right)$ to destroy $\beta$. Specifically, no foliation carried by the original branched surface $W$ with an integral curve over $\beta$ is carried by the new branched surface $W^{\prime}$.

So given any critical branching arc $\beta \in \Gamma$, we may modify $W$ to obtain a new branched surface $W^{\prime}$, also generated by disks and carrying $F$, such that any foliation carried by $\widehat{W}$ and containing nonseparable leaves linked by a lift $\widehat{\beta}$ of $\beta$ is not carried by $\widehat{W}^{\prime}$. By applying Lemma A [Go-Sh], we see that $W^{\prime}$ also has a transitive dual and so is stably taut. Furthermore, every foliation carried by $W^{\prime}$ is carried by $W$, since $N(W)$ can be obtained from $N\left(W^{\prime}\right)$ by pinching together a component of $\partial N\left(W^{\prime}\right)$ (contained in a branched leaf of the foliation). This amounts to removing a disk from the generating set for $W^{\prime}$ to obtain the original generating set $\Delta$ for $W$.

By hypothesis, the set $\Gamma=\left\{\beta_{1}, \ldots, \beta_{n}\right\}$ is finite and the lift of any non$\mathbf{R}$-covered foliation carried by $W$ to a foliation carried by $\widehat{W}$ contains a pair of nonseparable leaves linked by a lift of some arc in $\Gamma$. Moreover, we may assume each arc in $\Gamma$ is critical. In particular, we can modify $W$ as above to either destroy $\beta_{1}$ or create a bypass for it. We do so in a way that splits $N(W)$ along a curve meeting each $\beta_{i}, i \neq 1$, either transversely at interior points or not at all. In this sense, the new branched surface $W_{1}$ contains a branching arc corresponding to $\beta_{i}$ for every $i>1$. Furthermore, the lift of 
any non-R-covered foliation carried by $W_{1}$ contains a pair of leaves linked by a lift of one such arc.

We next apply the same technique to either destroy the branching arc in $W_{1}$ corresponding to $\beta_{2}$ or create a bypass for it; this yields a new branched surface $W_{2}$. Continuing in this manner, we obtain a finite string of branched surfaces $W=W_{0}, W_{1}, \ldots, W_{n}$, each carrying $F$. Let $W^{\prime}=W_{n}$.

Now suppose there is a foliation $G$ carried by $W^{\prime}$ whose lift $\widehat{G}$ to the universal cover has a non-Hausdorff leaf space. The foliation $G$ is also carried by $W$, so for some $1 \leq i \leq n$, a lift $\widehat{\beta}_{i}$ of $\beta_{i}$ links nonseparable leaves $\widehat{A}$ and $\widehat{B}$ in $\widehat{G}$. However, $W_{i}$ was obtained by modifying $W_{i-1}$ to either destroy the branching arc (in $W_{i-1}$ ) corresponding to $\beta_{i}$ or create a bypass for it. Since some lift of this branching arc also links $\widehat{A}$ and $\widehat{B}$, the foliation $\widehat{G}$ is not carried by $\widehat{W}_{i}$, a contradiction.

It follows that every foliation carried by $W^{\prime}$ is $\mathbf{R}$-covered.

As noted above, any transversely orientable $\mathbf{R}$-covered foliation of a closed orientable 3-manifold $M \neq S^{2} \times S^{1}$ is carried by a stably taut branched surface. So the essential hypothesis of Theorem 2.2 is the existence of the set $\Gamma$. It is also worth mentioning that if we omit the assumption that disks generate $W$, the proof above can still be used to show $F$ is carried by an $\mathbf{R}$-covered branched surface $W^{\prime}$. The only thing that is lost is the guarantee that $W^{\prime}$ is stably $\mathbf{R}$-covered.

A negatively (positively) branching arc $\beta$ with no upper (lower respectively) branches along its interior is contained in the boundary of some component of $M-W$. In particular, $\beta$ is the projection of a smoothly immersed curve whose interior is contained in some element of the generating set $\Delta$ and whose endpoints are in the boundary of that generating surface. If $W$ is generated by disks and has all disk sectors, then there are at most finitely many such branching arcs in $W$ up to equivalence. In particular, we have the following:

Corollary 2.3. Given a stably taut branched surface $W$ with disk sectors constructed from an $\mathbf{R}$-covered foliation $F$ of a closed orientable 3-manifold $M$, let $\widehat{W}$ be the lift of $W$ to the universal cover $\widehat{M}$. If for every non$\mathbf{R}$-covered foliation carried by $W$ there is a branching arc contained in the boundary of some component of $\widehat{M}-\widehat{W}$ linking nonseparable leaves in the universal cover, then there exists a stably $\mathbf{R}$-covered branched surface $W^{\prime}$ carrying $F$ and the $\mathbf{R}$-covered property is stable for $F$.

\section{R-covered branched surfaces}

In this section, we find a condition on the branch set of a taut branched surface $W$ constructed from $\mathbf{R}$-covered foliation $F$ of a closed orientable manifold $M \neq S^{2} \times S^{1}$ that guarantees the existence of an $\mathbf{R}$-covered branched 
surface carrying $F$. It is always possible to ensure all sectors of $W$ are embedded disks, so throughout we assume this is the case. However, we shall not assume that disks generate $W$ unless this is explicitly stated. Since this means the map of $W(\widehat{W})$ into the ambient manifold $M$ ( $\widehat{M}$ respectively) by inclusion is not always $\pi_{1}$-injective, we assume the range of all homotopy maps is the branched surface unless otherwise stated.

An arc embedded in the branch set $\widehat{\mu}$ of $\widehat{W}$ with an upper (lower) outgoing sector branching from its initial point and an upper (lower respectively) incoming sector branching into its terminal point is called a branching arc in $\widehat{\mu}$. (Each branching arc in $\widehat{\mu}$ covers a branching arc in $\mu$.)

For example, if $W$ is a taut branched surface with disk sectors, then any branching arc $\widehat{\beta}$ in $\widehat{W}$ transverse to $\widehat{\mu}$ is homotopic to a curve containing such an arc, provided that it links nonseparable leaves $\widehat{A}$ and $\widehat{B}$ in the lift of some foliation $G$ carried by $W$. Specifically, there is, by definition, a smoothly embedded curve $\widehat{\kappa}$ in some leaf $\widehat{K}$ of $\widehat{G}$ that projects injectively onto $\widehat{\beta}$. It follows that $\widehat{\beta}$ is smoothly embedded in $\widehat{\pi}(\widehat{K})$ and there exists an arc immersed in $\widehat{\mu}$ that is piecewise homotopic to $\widehat{\beta}$ through disk sectors of $\widehat{W}$ contained in $\widehat{\pi}(\widehat{K})$. By Reeb stability [Re], each of these disk sectors is covered injectively by a disk embedded in $\widehat{K}$. So there is a curve $\widehat{\gamma}$ immersed in $\widehat{K}$ and homotopic to $\widehat{\kappa}$ whose projection $\widehat{\pi}(\widehat{\gamma})$ is a curve in $\widehat{\mu}$ homotopic to $\widehat{\beta}$. If $\widehat{\gamma}$ contains a loop, i.e., if there exist subarcs $\widehat{\gamma}_{1}$ and $\widehat{\gamma}_{2}$ of $\widehat{\gamma}$ and a closed subarc $\hat{\lambda}$ of $\widehat{\gamma}$ that begins and ends at the same point such that $\widehat{\gamma}$ can be written as a composition $\widehat{\gamma}_{1} * \widehat{\lambda} * \widehat{\gamma}_{2}$, then $\widehat{\lambda}$ is null homotopic in $\widehat{K}$. In this case, the curve $\widehat{\gamma}_{1} * \widehat{\gamma}_{2}$, obtained by removing $\hat{\lambda}$ from $\widehat{\gamma}$ and reparameterizing the remaining arcs, $\widehat{\gamma}_{1}$ and $\widehat{\gamma}_{2}$, is also homotopic to $\widehat{\kappa}$ in $\widehat{K}$. So we may assume $\widehat{\gamma}$ contains no loops. It follows that $\widehat{\pi}(\widehat{\gamma})$ contains no loops, since otherwise there are two distinct points $\widehat{x}$ and $\widehat{y}$ in $\widehat{\gamma}$ that project onto the same point of $\widehat{W}$. However, if this occurs, we could construct a closed curve by taking the composition of a curve in $\widehat{K}$ joining $\widehat{x}$ and $\widehat{y}$ with a curve contained in a fiber of $N(\widehat{W})$ up to orientation. Modifying this curve slightly, we could then produce a closed loop transverse to $\widehat{G}$ that is null homotopic in $\widehat{M}$, contradicting our assumption that every foliation carried by $W$ is taut. Now since $\widehat{\pi}(\widehat{\gamma})$ begins and ends in $\widehat{\pi}(\widehat{A})$ and $\widehat{\pi}(\widehat{B})$ respectively, it has a subarc $\widehat{\beta}_{\mu}$ which is also embedded in $\widehat{\mu}$ and whose ends both branch into the negative (or positive) side of two smooth local subsets of $\widehat{\pi}(\widehat{A})$ and $\widehat{\pi}(\widehat{B})$ respectively. Furthermore, there is a curve embedded in $\widehat{K}$ that lies over $\widehat{\beta}_{\mu}$ in $N(\widehat{W})$; namely the corresponding subcurve of $\widehat{\gamma}$. An arc $\widehat{\beta}_{\mu}$ with these properties is called a critical branching arc in $\widehat{\mu}$ linking $\widehat{A}$ and $\widehat{B}$. Its quotient $\beta_{\mu}$ is a critical branching arc in $\mu$. Since $\widehat{\beta}_{\mu}$ contains 
no loops that are null homotopic in $\widehat{W}$, the curve $\beta_{\mu}$ contains no loops that are null homotopic in $W$.

It is worth noting that branching arcs in the branch set are not necessarily smooth. Moreover, a branching arc in $\widehat{\mu}$ with $\widehat{\pi}(\widehat{A})$ and $\widehat{\pi}(\widehat{B})$ branching from its respective ends is not necessarily critical, since to link $\widehat{A}$ and $\widehat{B}$ the arc must lie in a smoothly embedded plane in $\widehat{W}$. It is also worth noting that there seems to be no natural way to extend the notion of equivalence to branching arcs in $\widehat{\mu}$.

A bypass for a negatively (positively) branching arc in $\widehat{\mu}$ will be a homotopic curve, either embedded in $\widehat{\mu}$ or smoothly embedded in $\widehat{W}$ and transverse to $\widehat{\mu}$, that contains no negatively (positively) branching arcs. Its quotient in $W$ is a bypass for the corresponding branching arc in $\mu$. We say a finite arc $\tau$ embedded in $\mu$ is inescapable if every upper (lower) outgoing sector along $\tau$ branches from some negatively (positively) branching arc that is both contained in $\tau$ and has a bypass with no upper (lower, respectively) outgoing sectors. A finite arc $\widehat{\tau}$ embedded in $\widehat{\mu}$ is inescapable if it is the lift of an inescapable arc in $\mu$.

Lemma 3.1. Given a branched surface $W$ constructed from a foliation $F$ of a closed orientable 3-manifold $M$, let $\widehat{F}$ and $\widehat{W}$ be the lifts of $F$ and $W$, respectively, to the universal cover. If the projection $\widehat{\pi}(\widehat{L})$ of some leaf $\widehat{L}$ of $\widehat{F}$ meets the initial point of an inescapable curve $\widehat{\tau}$ in the branch set $\widehat{\mu}$ of $\widehat{W}$, then $\widehat{\pi}(\widehat{L})$ also meets the terminal point of $\widehat{\tau}$.

Proof. Suppose $\widehat{\tau}(t)_{0 \leq t \leq 1}$ is an inescapable curve embedded in the branch set $\widehat{\mu}$ of $\widehat{W}$. Let $\widehat{L}$ be a leaf in a foliation $\widehat{F}$ carried by $\widehat{W}$ such that its projection $\widehat{\pi}(\widehat{L})$ meets $\widehat{\tau}(0)$. Any time $\widehat{\pi}(\widehat{L})$ branches away from $\widehat{\tau}$, it does so along an outgoing sector at a crossing of $\widehat{\mu}$ contained in $\widehat{\tau}$. By our construction of $W$ and $\widehat{W}$, crossings of $\widehat{\mu}$ cannot accumulate in the lift of an evenly covered neighborhood of $W$. So since $\widehat{\tau}$ is compact, it contains only finitely many crossings. As a consequence, if $\widehat{\pi}(\widehat{L})$ does not meet $\widehat{\tau}(1)$, there is a last point $\widehat{\tau}\left(t^{\prime}\right)$ along $\widehat{\tau}$ that is contained in $\widehat{\pi}(\widehat{L})$.

In this case, $\widehat{\pi}(\widehat{L})$ branches away from $\widehat{\tau}$ along, say, an upper outgoing sector at $\widehat{\tau}\left(t^{\prime}\right), 0 \leq t^{\prime}<1$. By hypothesis, there exists $t_{0} \leq t^{\prime} \leq t_{1}$, $t_{0} \neq t_{1}$, such that $\widehat{\tau}(t)_{t_{0} \leq t \leq t_{1}}$ is a negatively branching arc. Furthermore, this branching arc has a bypass $\widehat{\delta}$ with no upper outgoing sectors branching from it. So there is an embedded curve $\widehat{\eta}$ over $\widehat{\delta}$, beginning and ending at the uppermost points over $\widehat{\tau}\left(t_{0}\right)$ and $\widehat{\tau}\left(t_{1}\right)$ respectively, which is a composition of integral curves of $\widehat{F}$ and positive transversals contained in fibers of $N(\widehat{W})$. Furthermore, we can find an embedded curve $\widehat{\gamma}(t)_{t_{0} \leq t \leq t_{1}}$ where $\widehat{\gamma}(t)_{t_{0} \leq t \leq t^{\prime}}$ lies over $\widehat{\tau}(t)_{t_{0} \leq t \leq t^{\prime}}$ and is contained in a leaf below $\widehat{L}$, and $\widehat{\gamma}(t)_{t^{\prime} \leq t \leq t_{1}}$ lies over $\widehat{\tau}(t)_{t^{\prime} \leq t \leq t_{1}}$ and consists of integral curves of $\widehat{F}$ and transversals to $\widehat{F}$ 


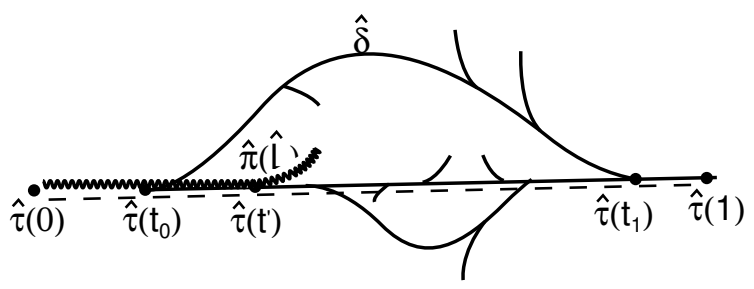

Indicates $\widehat{\tau}$ and indicates $\widehat{\pi}(\widehat{L})$.

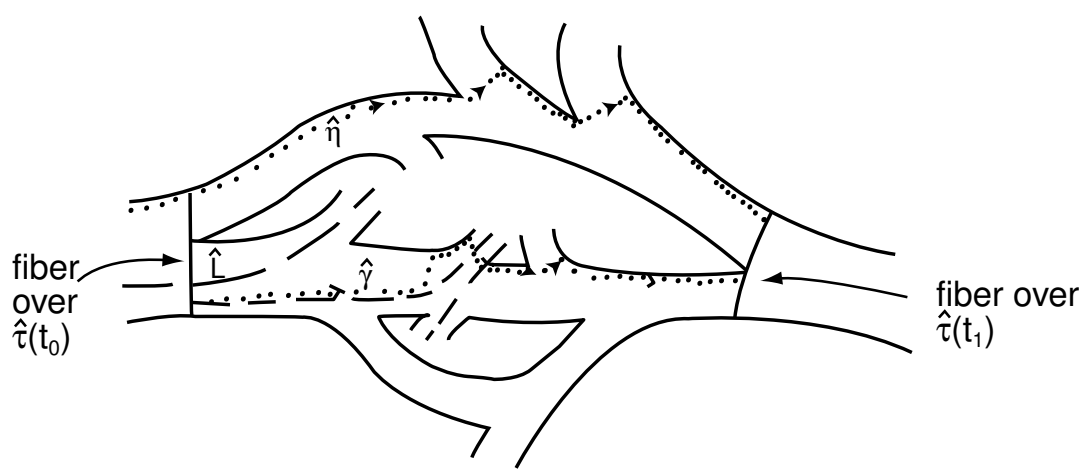

Indicates leaves of $\widehat{F}$ and indicates $\widehat{\eta}$ and $\widehat{\gamma}$.

Figure 3.1.

contained in fibers of $N(\widehat{W})$ up to orientation. See Figure 3.1. We then have a disk in $\widehat{M}$ bounded by $\widehat{\gamma}, \widehat{\eta}$, and transversals over $\widehat{\tau}\left(t_{0}\right)$ and $\widehat{\tau}\left(t_{1}\right)$ respectively which can be put in general position with respect to the foliation $\widehat{F}$. The leaf $\widehat{L}$ meets the boundary of this disk transversely at some point over $\widehat{\tau}\left(t_{0}\right)$. Furthermore, $\widehat{L}$ cannot cross any of the positive transversals in $\widehat{\eta}$ since $\widehat{F}$ is transversely orientable. So $\widehat{L}$ must cross either $\widehat{\gamma}(t)_{t^{\prime} \leq t \leq t_{1}}$ or the transversal over $\widehat{\tau}\left(t_{1}\right)$. In both cases, we have a contradiction to the way we chose $t^{\prime}$. So $\widehat{\pi}(\widehat{L})$ contains $\widehat{\tau}(1)$.

In what follows, a parameterized embedded copy of $S^{1}$ in $\mu$ that does not bound an embedded disk in $W$ will be called an essential loop. Furthermore, we shall say a branching arc in $\mu$ is elementary if it contains no loops.

The following theorem offers a verifiable condition on the branch set of a branched surface carrying an $\mathbf{R}$-covered foliation $F$ that guarantees the existence of an $\mathbf{R}$-covered branched surface carrying $F$.

Theorem 3.2. Given a taut branched surface $W$ with disk sectors constructed from a foliation of a closed orientable 3 -manifold $M \neq S^{2} \times S^{1}$, let $\alpha(t)_{0 \leq t \leq 1}$ be an arc embedded in the branch set $\mu$ that has an outgoing 
sector branching from its initial point and contains no loops. Suppose that for every such $\alpha$ and every essential loop $\lambda$ in $\mu$ based at $\alpha(1)$, there exist homotopic curves $\eta \subseteq \alpha * \lambda$ and $\tau \subseteq \mu$ beginning at $\alpha(0)$ such that $\eta$ has nonempty interior and $\tau$ is inescapable. For any $\mathbf{R}$-covered foliation $F$ carried by $W$, there exists an $\mathbf{R}$-covered branched surface $W^{\prime}$ also carrying $F$; in particular, when $W$ is generated by disks, the $\mathbf{R}$-covered property is stable for $F$.

Proof. Suppose $G$ is a non-R-covered foliation carried by $W$ and let $\widehat{\beta}$ be a critical branching arc in the branch set $\widehat{\mu}$ of $\widehat{W}$ linking nonseparable leaves $\widehat{A}$ and $\widehat{B}$ of $\widehat{G}$. We may choose $\widehat{\beta}$ so that it contains no other branching arc linking $\widehat{A}$ and $\widehat{B}$. We may also assume, without loss of generality, that $\widehat{A}$ and $\widehat{B}$ are nonseparable on their negative sides, so $\widehat{\beta}$ is negatively branching. By definition, $\widehat{\beta}$ is the projection of an integral curve of $\widehat{G}$ onto $\widehat{W}$. As noted above, it descends to a branching arc $\beta$ in $\mu$ that contains no loops which are null homotopic in $W$ (i.e., there is no subarc of $\beta$ with nonempty interior that begins and ends at the same point and is null homotopic).

So if $\beta$ contains a loop, then it contains an essential loop. In this case, let $\lambda$ be the first essential loop in $\beta$ and let $\alpha$ be the arc in $\beta$ from its initial point $\beta(0)$ to the initial point of $\lambda$. We may now choose homotopic curves $\eta$ in $\alpha * \lambda$ and $\tau$ in $\mu$ as in the hypotheses. Since $\tau$ is inescapable it is, by definition, embedded in $\mu$. So $\tau$ lifts to a curve $\widehat{\tau}$ embedded in $\widehat{\mu}$ with the same initial point as $\widehat{\beta}$. In particular, $\widehat{\tau}$ begins in $\widehat{\pi}(\widehat{A})$ and, by Lemma 3.1 , $\widehat{\tau}$ ends in $\widehat{\pi}(\widehat{A}) \cap \operatorname{int}(\widehat{\beta})$. By assumption, $\widehat{B}$ meets the fiber over $\widehat{\beta}(1)$, so $\widehat{A}$ cannot meet this fiber. Therefore, $\widehat{\pi}(\widehat{A})$ must branch away from $\operatorname{int}(\widehat{\beta})$ along an upper sector, a contradiction since we chose $\widehat{\beta}$ to contain no other critical branching arcs linking $\widehat{A}$ and $\widehat{B}$.

It follows that any pair of nonseparable leaves $\widehat{A}$ and $\widehat{B}$ in $\widehat{G}$ is linked by a critical branching arc $\widehat{\beta}$ in $\mu$ which descends to an elementary branching arc $\beta$ in $\mu$. Furthermore, $\beta$ can be perturbed slightly onto adjacent sectors to obtain a homotopic branching arc that is nowhere tangent to $\mu$, contains no crossings of $\mu$ and also lifts to a branching arc linking $\widehat{A}$ and $\widehat{B}$. Now for every elementary branching arc $\beta$ in $\mu$, there are at most finitely many homotopic branching arcs (up to equivalence) that can be obtained by perturbing $\beta$ in this manner. Since there are at most finitely many elementary branching arcs in $\mu$, the result now follows from the remark after Theorem 2.2.

As above, let $W$ be a taut branched surface with disk sectors and let $F$ be an $\mathbf{R}$-covered foliation carried by $W$. We say a negatively (positively) branching arc in the branch set $\mu$ of $W$ is simple if it is elementary and has no upper (lower, respectively) branches along its interior. Clearly there are at most finitely many simple branching arcs in $\mu$ and each is contained in the boundary of some component of $M-W$. In particular, each simple 
branching arc $\beta$ is the projection of an integral curve of $F$. So we can split $N(W)$ along disks embedded in leaves of $F$, as in the proof of Theorem 2.2, to create bypasses for all the simple branching arcs in $\mu$. (For each simple negatively (positively) branching arc $\beta$, the disk we use is met by forward (backward) orbits of an integral curve $\gamma_{F}$ of $F$ which meets the uppermost (lowermost) point over one end of $\beta$ and also meets the fiber over the other end.) The branched surface we obtain, $V$, carries only foliations carried by $W$, including $F$. (If the lift of each non-R-covered foliation carried by $W$ contains a pair of nonseparable leaves linked by a simple branching arc in $\widehat{\mu}$, then the branched surface $V$ is $\mathbf{R}$-covered.)

Now, we can choose an orientation for each simple negatively (positively) branching arc $\beta$ in $\mu$ so that for any foliation carried by $\widehat{V}$, the leaves meeting the fiber of $N(\widehat{W})$ over $\widehat{\beta}(0)$ either meet the fiber over $\widehat{\beta}(1)$ or branch away from $\widehat{\beta}$ along a lower (upper respectively) outgoing sector. (For example, if $\beta$ is negatively branching, the orientation is chosen so that $\widehat{\gamma}_{F}$ is contained in the uppermost leaf of $\widehat{F}$ over $\widehat{\beta}(0)$; if some leaf of $\widehat{F}$ meets the uppermost point over both ends of $\widehat{\beta}$, then either orientation will suffice.) Then any curve $\tau$ in the branch set of $W$ yields an inescapable curve in $V$ if every upper (lower) outgoing sector branches from a negatively (positively) branching arc contained in $\tau$ that either has a bypass with no upper (lower respectively) outgoing sectors or is simple and oriented as above. So the $\tau$ in Theorem 3.2 need not be inescapable; rather, it need only satisfy this weaker condition. In other words, it is not necessary to explicitly construct $V$ from $W$ to check whether it satisfies the hypotheses of Theorem 3.2.

For example, let $G_{0}$ be a foliation of $T^{2}$ with two unstable Reeb components, and consider a foliation $G$ of $T^{2} \times S^{1}$ where each leaf is the product of a leaf in $G_{0}$ with an $S_{1}$ fiber. Clearly an $\mathbf{R}$-covered foliation $F$ of $T^{2} \times S^{1}$ can be chosen arbitrarily close to $G$. We construct a branched surface carrying both $F$ and $G$ as follows:

Begin by choosing two annuli $A_{1}$ and $A_{2}$, contained in leaves of both $F$ and $G$, as shown in Figure 3.2. (We assume the figure is contained in a copy of $[0,1] \times[0,1] \times[0,1]$ where opposite horizontal and vertical sides are identified in the natural way to obtain $T^{2} \times S^{1}$.) Orbits of a flow $\phi$ transverse to both $F$ and $G$ are indicated by the oriented dashed curves. The generating set $\left\{A_{1}, A_{2}\right\}$ together with the flow $\phi$ can be used to construct the branched surface $W$ shown in Figure 3.3. Note that this branched surface does not have disk sectors and its branch set is not connected. However, we modify it so that it has these desired properties by splitting along two disks $D_{1}$ and $D_{2}$ embedded in $W$ as shown in Figure 3.4(a), and enlarging the two components in the complement of $W$. The latter type of modification involves splitting $W$ along strips $V_{1}$ and $V_{2}$, like those shown in Figure 3.4(b), that do not intersect the disks $D_{1}$ and $D_{2}$. (We can think this as "sticking our finger" 


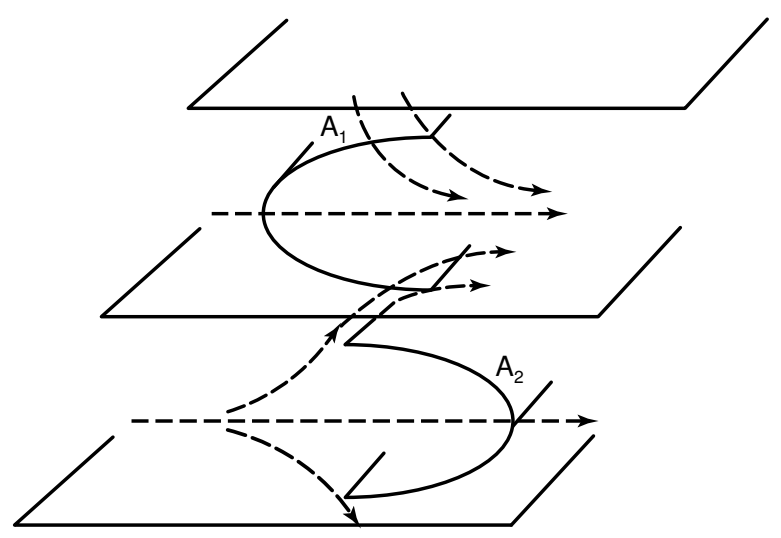

Figure 3.2.

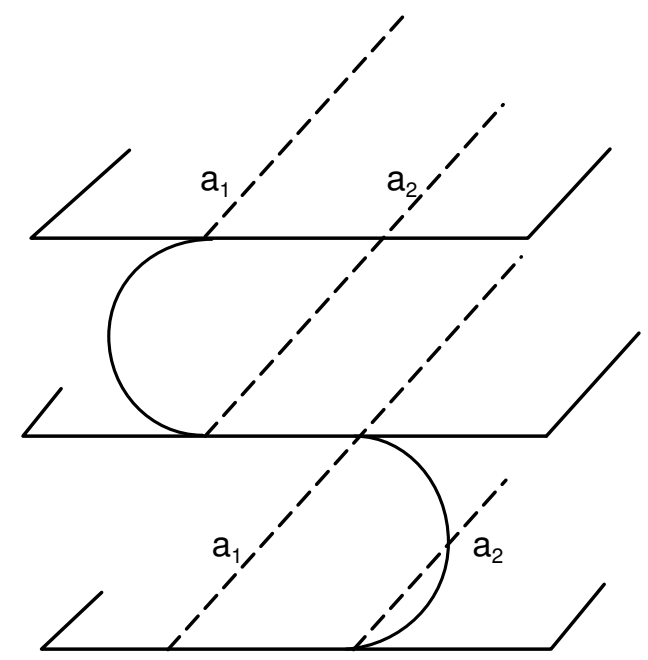

Figure 3.3. Labels indicate closed curves in the branch set that are identified.

into the branch set so that it meets the boundary of one of these strips $V_{i}$ and pushing it into the one-sheeted side at this branching so that it separates the branched surface along $V_{i}$.) Each of these splittings corresponds to a modification of the original generating set. The splittings along $D_{1}$ and $D_{2}$ are the result of adding two disks to the original generating set, and the splittings along $V_{1}$ and $V_{2}$ are the result of enlarging $A_{1}$ and $A_{2}$ respectively. See [Sh1] for details.

Figure 3.5 shows the branch set for the resulting $W$. The 3 -sheeted side of each crossing of $\mu$ has been labeled $R$ or $L$ to distinguish between the two possible local neighborhoods in Figures 1.1(c) and 1.1(d), respectively. 


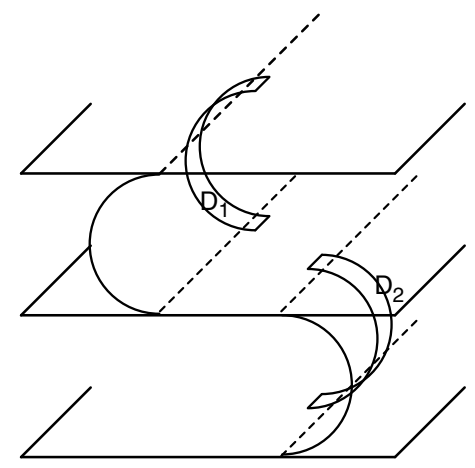

(a)

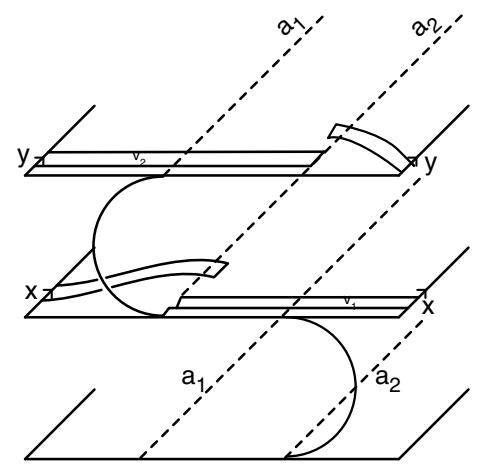

(b)

Figure 3.4. Labels indicate arcs in the boundaries of $V_{1}$ and $V_{2}$ that are identified.

We have labeled the two arcs bounding the 3 -sheeted side of each crossing with + or - to indicate an upper outgoing sector or a lower outgoing sector, respectively, as we leave the crossing along that arc. The labels make it easy to identify the negative and positive branching arcs in $\mu$. However, determining whether a branching arc has a bypass and finding such a bypass if one does exist, requires that we visualize how the splittings described above change the original branched surface in Figure 3.3. For example, the six elementary branching arcs indicated by the dotted lines in Figure 3.5 are each contained in the image of either $V_{1}$ or $V_{2}$ after the splittings. Figure 3.6 shows three copies of the image of $V_{2}$ in $W$. In each, a curve corresponding to one of the three branching arcs in Figure 3.5 labeled $\lambda_{1}, \lambda_{2}$ and $\lambda_{3}$ is indicated. Clearly each of these branching arcs has a bypass with no branches along its interior.

Next consider the eight remaining simple branching arcs in $\mu$. Each lies in the boundary of either $D_{1}$ or $D_{2}$. Precisely four of these branching arcs, indicated by the dashed lines, have no bypass. (The other four each have a bypass whose interior lies in a sector of $W$.) However, we can split $N(W)$ along disks in leaves of $F$, as described above, to create bypasses for these branching arcs. For each of the two positively branching arcs, the disk we use is met by the backward orbits of an integral curve $\gamma_{F}$ which begins and ends with the lowermost points of the fibers over the respective ends of that branching arc. Likewise, the disk we use for each of the two negatively branching arcs is met by forward orbits of an integral curve $\gamma_{F}$ which begins and ends with the uppermost points of the fibers over both ends of the branching arc.

It is now straightforward to verify that for every $\alpha$ and $\lambda$ as in the hypotheses of Theorem 3.2, a subarc $\eta$ of $\alpha * \lambda$ can be found homotopic to some curve $\tau$ embedded in $\mu$ with the property that every upper (lower) 


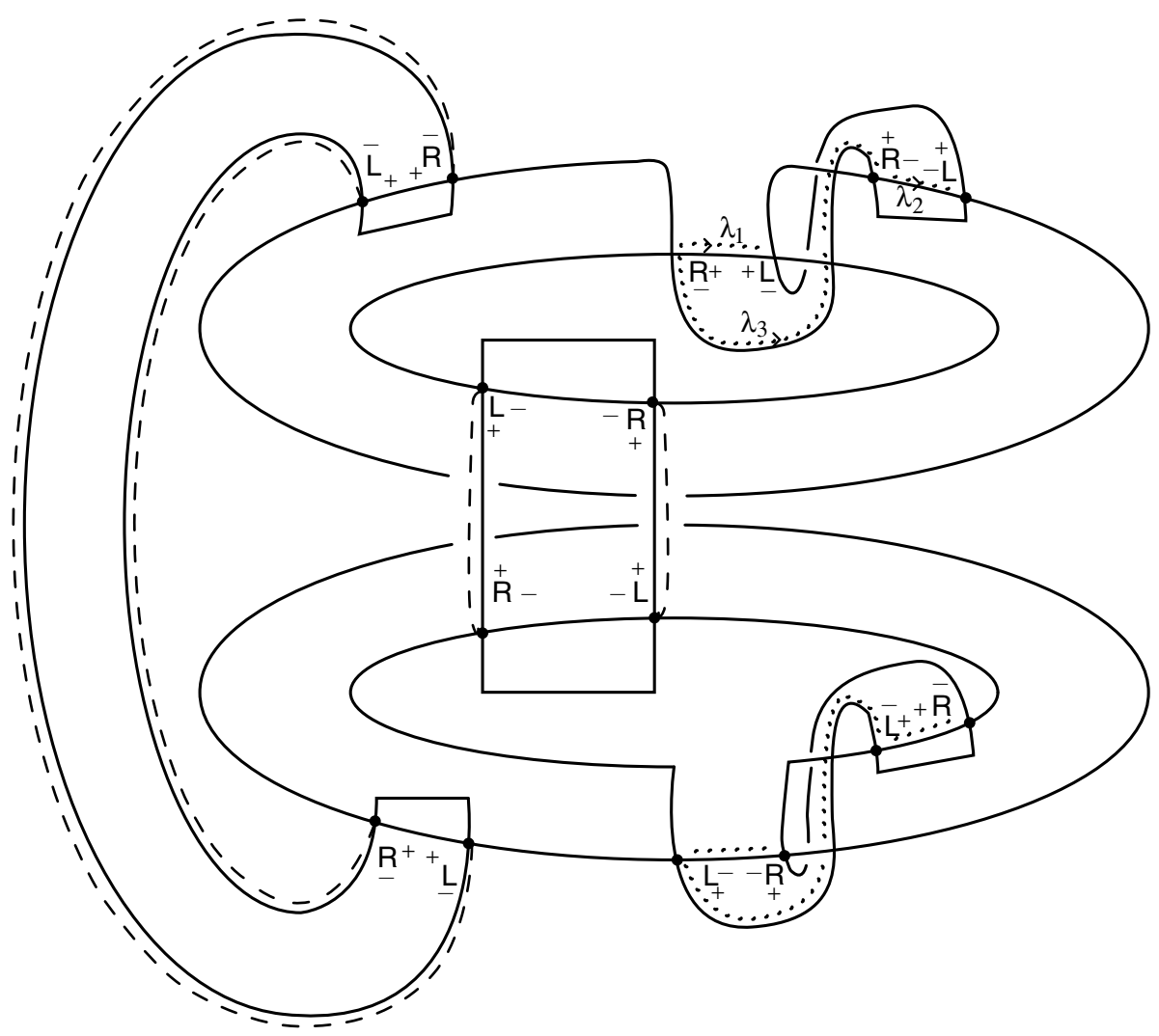

Figure 3.5.
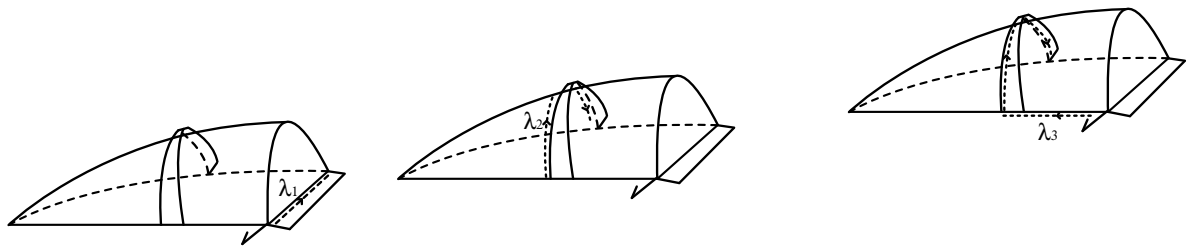

Figure 3.6.

outgoing sector along $\tau$ branches from a negatively (positively) branching arc in $\tau$ that either has a bypass with no upper (lower respectively) outgoing sectors or is simple. Consequently, $W$ can be modified to obtain an $\mathbf{R}$-covered branched surface.

There are, of course, branched surfaces carrying $\mathbf{R}$-covered foliations that do not satisfy the hypotheses of Theorem 3.2. For example, let $M=\Sigma_{2} \times S^{1}$, where $\Sigma_{2}$ is the compact surface of genus two. Figure 3.7 shows a projection $p(\mu)$ of the branch set $\mu$ for a stably taut branched surface $W$, constructed 


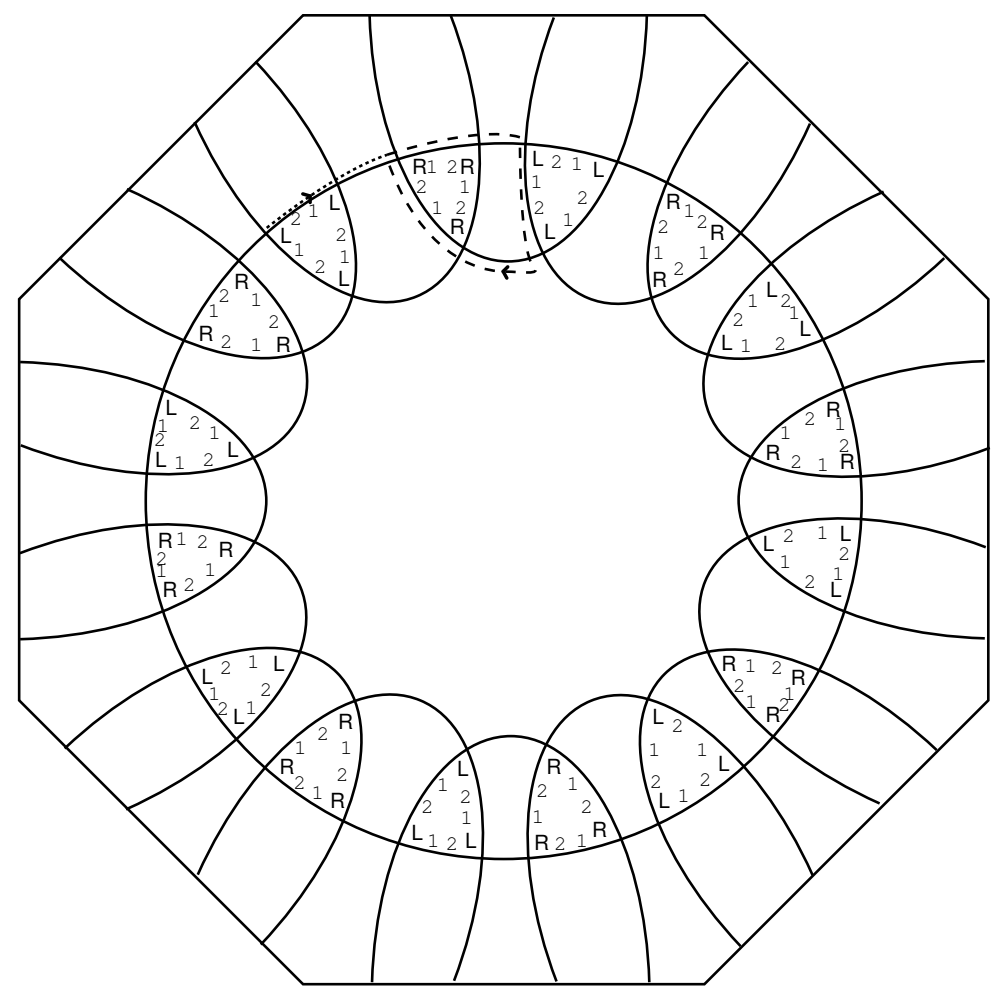

Figure 3.7.

from the trivial foliation $F$ of $M$ by compact surfaces transverse to the $S^{1}$ fibers, onto the base space $\Sigma_{2}$. (This projection $p(\mu)$ is shown embedded in a planar model of $\Sigma_{2}$, where we are assuming opposite sides of the octagon are identified.) By the way we chose the generating disks, $p(\mu)$ is symmetric under both horizontal and vertical reflection of Figure 3.7 and under forty five degree clockwise and counterclockwise rotation of this planar model about its center.

In particular, the generating set for $W$ consists of six disks chosen in three different leaves (so $W$ carries all foliations sufficiently close to $F$ ). The transverse flow $\phi$ used for the construction is orthogonal to the foliation $F$, so its orbits are closed and contained in the $S^{1}$ fibers. Consequently, the boundary of each generating disk yields a smooth embedded copy of $S^{1}$ in the branch set. For example, Figure 3.8(a) shows projected parts of four generating disks $D_{1}, D_{2}, D_{3}$ and $D_{4}$. Each boundary point marked with an "X" yields a crossing of $W$. The remaining portions of the disk boundaries have been labeled 1,2, 3 or 4 to indicate whether the points in this portion flow forward under $\phi$ into $D_{1}, D_{2}, D_{3}$ or $D_{4}$, respectively, before meeting another disk. Figure 3.8(b) shows the corresponding piece of $p(\mu)$. We 

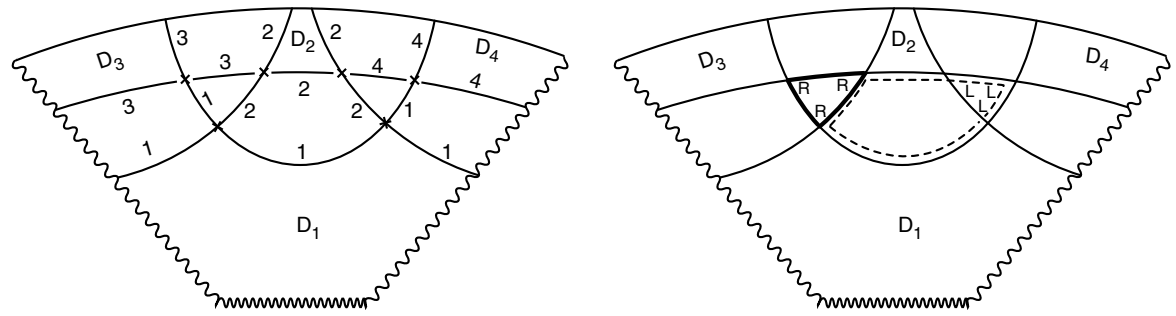

Figure 3.8. Left: (a), right: (b).

see, for example, that the two loops indicated by the dashed and bold lines respectively correspond to essential loops in $W$ which, up to orientation, are homotopic to $S^{1}$ fibers. (There are many other essential loops in $\mu$.)

Now, one can construct non-R-covered foliations arbitrarily close to the foliation $F([\mathbf{C a}],[\mathbf{F e} 5])$ so, as discussed in Section $2, W$ cannot be stably $\mathbf{R}$-covered. Indeed, if we choose $\lambda$ to be the essential loop indicated by the dashed curve in Figure 3.7 and let $\alpha$ be the embedded arc indicated by the dotted line, it is straightforward to check that there are no corresponding curves $\eta$ and $\tau$ as in the hypotheses of Theorem 3.2. (As in Figure 3.5, we have labeled the two arcs bounding the 3-sheeted side of each crossing with + or - to indicate an upper outgoing sector or a lower outgoing sector, respectively, as we leave the crossing along that arc.)

Finally, we note that for Theorem 3.2 we need only consider $\alpha$ and $\lambda$ for which $\alpha * \lambda$ is contained in the projection of a surface smoothly embedded in $N(W)$ and transverse to the fibers. (This is necessary for a branching arc $\beta$ containing $\alpha * \lambda$ to be critical.) In the previous example, the indicated curve $\alpha * \lambda$ satisfies this additional restriction. So, as we should expect, the hypotheses of Theorem 3.2 still fail to hold under these weaker conditions. It is, however, possible that an $\mathbf{R}$-covered branched surface carrying $F$ could be constructed using generating surfaces other than disks.

In fact, for many explicit examples of $\mathbf{R}$-covered foliations it is possible to construct a branched surface that satisfies the hypotheses of either Theorem 2.2 or Theorem 3.2 if we allow the generating set to contain surfaces other than disks. At this stage, we cannot claim that any $\mathbf{R}$-covered foliation is carried by some $\mathbf{R}$-covered branched surface, although we conjecture that it is so. On the other hand, not every $\mathbf{R}$-covered foliation is carried by a stably $\mathbf{R}$-covered branched surface, since there are examples of non$\mathbf{R}$-covered foliations arbitrarily near $\mathbf{R}$-covered foliations. However, it is possible that every $\mathbf{R}$-covered foliation for which the $\mathbf{R}$-covered property is stable is carried by a stably $\mathbf{R}$-covered branched surface.

\section{References}

[An] D.V. Anosov, Geodesic flows on closed Riemannian manifolds with negative curvature, Proc. Steklov Inst. Math., 90, 1969, MR 0242194 (39 \#3527). 
[Ba1] T. Barbot, Géométrie transverse des flots d'Anosov, Thesis, Ecole Norm. Sup., Lyon, 1992.

[Ba2] T. Barbot, Caractérisation des flots d'Anosov en dimension 3 par leurs feuilletages faibles, Ergodic Theory Dynam. Systems, 15 (1995), 247-470, MR 1332403 (96d:58100), Zbl 0826.58025.

[Bo-La] C. Bonatti and R. Langevin, Un example de flot d'Anosov transitif transverse à un tore et non conjugué à une suspension, Ergodic Theory Dynam. Systems, 14 (1994), 633-643, MR 1304136 (95j:58129), Zbl 0826.58026.

[Ca] D. Calegari, R-covered foliations of hyperbolic 3-manifolds, Geometry and Topology, 3 (1999), 137-153, MR 1695533 (2000c:57038), Zbl 0924.57014.

[Ch-Go] J. Christy and S. Goodman, Branched surfaces transverse to codimension one foliations, preprint.

[Fe1] S. Fenley, Anosov flows in 3-manifolds, Ann. of Math., 139 (1994), 79-115, MR 1259365 (94m:58162), Zbl 0796.58039.

[Fe2] S. Fenley, Continuous extension of Anosov foliations in 3-manifolds with negatively curved fundamental group, Pacific J. Math., 186 (1998), 201-216, MR 1663798 (99k:58135), Zbl 1012.37018.

[Fe3] S. Fenley, Quasi-isometric foliations, Topology, 31 (1992), 667-676, MR 1174265 (94a:57044), Zbl 0770.57015.

[Fe4] S. Fenley, The structure of branching in the Anosov flows of 3-manifolds, Comment. Math. Helv., 73 (1998), 259-297, MR 1611703 (99a:58123), Zbl 0999.37008.

[Fe5] S. Fenley, Regulating flow, topology and rigidity, preprint, 2000.

[Fe6] S. Fenley, Asymptotic properties of depth one foliations in hyperbolic 3manifolds, J. Differential Geom., 36 (1992), 269-313, MR 1180384 (93k:57030), Zbl 0766.53018.

[Fr-Wi] J. Franks and R. Williams, Anomalous Anosov flows, Common Theory of Dynamical Systems, Lecture Notes in Mathematics, 819, Springer, Berlin, 1980, 158-174, MR 0591182 (82e:58078), Zbl 0463.58021.

[Ga1] D. Gabai, Foliations and the topology of 3-manifolds, J. Differential Geom., 18 (1983), 445-503, MR 0723813 (86a:57009), Zbl 0533.57013.

[Ga2] D. Gabai, Foliations and the topology of 3-manifolds, II, J. Differential Geom., 26 (1987), 461-478, MR 0910017 (89a:57014a), Zbl 0627.57012.

[Ga3] D. Gabai, Foliations and the topology of 3-manifolds, III, J. Differential Geom., 26 (1987), 479-536, MR 0910018 (89a:57014b), Zbl 0639.57008.

[Gh] E. Ghys, Flots d'Anosov sur les 3-variétés fibrés en cercles, Ergodic Theory Dynam. Systems, 4 (1984), 67-80, MR 0758894 (86b:58098), Zbl 0527.58030.

[Go-Sh] S. Goodman and S. Shields, A condition for the stability of $\mathbf{R}$-covered on foliations of 3-manifolds, Trans. Amer. Math. Soc., 352 (2000), 4051-4065, MR 1624178 (2000m:57042), Zbl 0965.57026.

[Ha] A. Haefliger, Varietés (non separées) à une dimension et structures feulletées du plan, L'enseignement Math., 3 (1957), 107-125, MR 0089412 (19,671c), Zbl 0079.17101.

[Hi] M.W. Hirsch, Stability of compact leaves of foliations, in 'Dynamical Systems' (Proc. Sympos., Univ. Bahia, Salvador, 1971), Academic Press, 1973, 135-153, MR 0334236 (48 \#12555), Zbl 0272.57015. 
[Im] H. Imanishi, On the theorem of Denjoy-Sacksteder for codimension one foliations without holonomy, J. Math. Kyoto Univ., 14 (1974), 607-634, MR 0368028 (51 \#4270), Zbl 0296.57006.

[No] S.P. Novikov, Topology of foliations, Trans. Moscow Math. Soc., 14 (1965), 248-278 (Russian); A.M.S. Translation, 1967, 268-304, MR 0200938 (34 \#824), Zbl 0247.57006.

[Pa] F. Palmeira, Open manifolds foliated by planes, Ann. of Math., 107 (1978), 109131, MR 0501018 (58 \#18490), Zbl 0382.57010.

[Pl1] J. Plante, Anosov flows, transversely affine foliations and a conjecture of Verjovsky, J. London Math. Soc., 23 (1981), 359-362, MR 0609116 (82g:58069), Zbl 0465.58020.

[P12] J. Plante, Solvable groups acting on the line, Trans. Amer. Math. Soc., 278 (1983), 401-414, MR 0697084 (85b:57048), Zbl 0569.57012.

[Re] G. Reeb, Sur certaines propriétés topologiques des variétés feuilletées, Publ. Inst. Math. Univ. Strasbourg, 11, 5-89, 155-156, Actualités Sci. Ind., 1183, Hermann, Paris, 1952, MR 0055692 (14,1113a).

[Ro] R. Roussarie, Plongements dans les variétés feuilletées et classification de feulletages sans holonomie, I.H.E.S. Sci. Publ. Math., 43 (1973), 101-142, MR 0358809 (50 \#11268), Zbl 0356.57017.

[Sh1] S. Shields, The stability of foliations of orientable 3-manifolds covered by a product, Trans. Amer. Math. Soc., 348 (1996), 4653-4671, MR 1355076 (97a:57019), Zbl 0869.57003.

[Sh2] S. Shields, Branched Surfaces and the Stability of Compact Leaves, Thesis, University of North Carolina at Chapel Hill, 1991.

[Sh3] S. Shields, Branching in the universal cover of taut foliations, Pacific J. Math., 203 (2002), 235-256, MR 1895932 (2003m:57061).

[So] V. Solodov, On the universal cover of Anosov flows, research announcement.

[Su] D. Sullivan, A homological characterization of foliations consisting of minimal surfaces, Comment. Math. Helv., 54 (1979), 218-223, MR 0535056 (80m:57022), Zbl 0409.57025.

[Th] W.P. Thurston, A norm for the homology of 3-manifolds, Mem. Amer. Math. Soc., 59(339) (1986), 99-130, MR 0823443 (88h:57014), Zbl 0585.57006.

[Wi] R.F. Williams, Expanding attractors, Inst. Hautes Études Sci. Publ. Math., 43 (1973), 169-203, MR 0348794 (50 \#1289), Zbl 0279.58013.

Received April 3, 2003 and revised August 8, 2003.

Department of Mathematics

College of Charleston

Charleston SC 29424-0001

E-mail address: shieldss@cofc.edu 